\title{
Inflectional Loci of Scrolls over Smooth, Projective Varieties
}

\author{
Antonio LANTERI, RaQUEl MallaVibarRena \& \\ RAGNI PIENE
}

\begin{abstract}
Let $X \subset \mathbb{P}^{N}$ be a scroll over an $m$-dimensional variety $Y$. We find the locally free sheaves on $X$ governing the osculating behavior of $X$, and, under certain dimension assumptions, we compute the cohomology class and the degree of the inflectional locus of $X$. The case $m=1$ was treated in [9]. Here we treat the case $m \geq 2$, which is more complicated for at least two reasons: the expression for the osculating sheaves and the computations of the class of the inflectional locus become more complex, and the dimension requirements needed to ensure validity of the formulas are more severe.
\end{abstract}

\section{INTRODUCTION}

Let $X \subset \mathbb{P}^{N}=\mathbb{P}(V)$ be a nondegenerate, smooth, complex projective variety of dimension $n$, let $\mathcal{L}$ denote the hyperplane bundle, and identify $V$ with a subspace of $H^{0}(X, \mathcal{L})$. Let $\mathcal{P}_{X}^{k}(\mathcal{L})$ be the $k$ th principal parts bundle of $\mathcal{L}$, and let $j_{k}: V_{X}=$ $V \otimes \mathcal{O}_{X} \rightarrow \mathcal{P}_{X}^{k}(\mathcal{L})$ be the sheaf homomorphism associating to every section $\sigma \in V$ its $k$ th jet evaluated at $x$, for every $x \in X$. The homomorphism $j_{k, x}$ allows us to define the $k$ th osculating space to $X$ at $x$ as follows: $\operatorname{Osc}_{x}^{k}(X):=\mathbb{P}\left(\operatorname{Im}\left(j_{k, x}\right)\right)$ (we interpret $\mathbb{P}(V)$ as the set of codimension 1 vector subspaces of $V$ ). Let $s_{k}$ denote the generic rank of $j_{k, x}$. The $k$ th inflectional locus $\Phi_{k}(X)$ of $X$ is defined to be the locus where the rank of $j_{k, x}$ is strictly less than $s_{k}$. In [9] we studied the 
particular case where $X$ is a scroll over a smooth curve. In this case the expected generic rank of $j_{k}$ is $k n+1$, for every $k$ such that $k n \leq N$. For the largest such $k$, we obtained an explicit formula for the integral cohomology class of $\Phi_{k}(X)$. In particular, it allowed us to count the flexes when there are finitely many and to characterize uninflected scrolls in an appropriate range.

When we consider scrolls $X$ over a higher-dimensional smooth projective variety $Y$, the situation becomes more difficult for several reasons. First of all, taking as a starting point the general framework of [9], the generalization of the construction of the osculating bundles when $m:=\operatorname{dim} Y \geq 2$ is not straightforward and requires the definition of a new bundle $\mathcal{T}_{k}$ to get the appropriate diagrams to determine the inflectional locus. Secondly, it becomes more and more difficult to obtain explicit expressions for the cohomology class of $\Phi_{k}(X)$, using Porteous's formula, as the dimension of $Y$ increases. As in the case of scrolls over curves [9], we need to impose that $k$ is such that $s_{k}$ is the maximum value allowed for a scroll of dimension $n$ over $Y$ in $\mathbb{P}^{N}$. This requirement on $m, n$, and $N$ fixes the range of validity of the results so that there are many examples that do not fulfill the requirement and that we therefore cannot treat with the present methods. Some of these examples, also interesting from the point of view of uninflectedness, are discussed in this paper. The dimension requirements are explained in Section 2, where we describe the general setting.

The main general results of the paper are Theorems 2.4 and 2.5. Theorem 2.4 provides the technical tools leading to a relevant exact diagram. With Theorem 2.5 we extract from this diagram a formal expression for the cohomology class of $\Phi_{k}(X)$, which is given by the product of $k+1$ inverses of the total Chern classes of some vector bundles involving symmetric powers of $T_{Y}$, the vector bundle $\pi_{*} \mathcal{L}$, where $\pi: X \rightarrow Y$ is the scroll projection, and $\mathcal{L}$ itself. In order to make this formula explicit, one needs either to simplify the vector bundles involved or to reduce the number of factors. The first approach is developed in Section 3, where we treat the case that $Y$ is an abelian variety. Here the complexity of the computation is represented only by the dimension $m$, and in this case we are able to get explicit formulas for the class of $\Phi_{k}(X)$ when $m=2$ (Proposition 3.1) and $m=3$ (Proposition 3.3). The second approach takes the rest of the paper. Here the complexity depends on the codimension $\ell$ of $\Phi_{2}(X)$. When $\ell=1$, in Section 4 we give an explicit formula for $\Phi_{2}(X)$ holding for any $m$ and $n$ (Theorem 4.1), a lower bound for its degree, and a better lower bound holding if a suitable adjoint bundle on $Y$ is nef. This situation is elaborated further in Section 5, assuming moreover that $m=2$ and $n=3$. In this case, for threefold scrolls in $\mathbb{P}^{8}$, Theorem 5.5 gives the lower bound $\operatorname{deg} \Phi_{2}(X) \geq 3 d$, where $d$ is the degree of $X$, with a few explicit exceptions. In the last two sections we take care of the case $\ell \geq 2$. In Section 6 we make explicit the expression of $\Phi_{2}(X)$ for $(n, m)=(3,2)$, and we discuss the uninflectedness of $X$ for $Y$ belonging to various classes of surfaces. Finally, in Section 7, explicit formulas for $\Phi_{2}(X)$ are computed, for various values of $\ell$, when $X$ is a four-dimensional scroll over a threefold $Y$, and we prove that for $Y=\mathbb{P}^{3}$ or $\mathbb{Q}^{3}$ none of these scrolls are uninflected. 


\section{SCROLLS}

Let $X \subset \mathbb{P}^{N}=\mathbb{P}(V)$ be a nondegenerate, smooth, $n$-dimensional scroll over a smooth $m$-dimensional variety $Y$, and let $\pi: X \rightarrow Y$ be the projection. We identify $V$ with a vector subspace of $H^{0}(X, \mathcal{L})$, where $\mathcal{L}$ is the hyperplane line bundle of $X$. Since $X$ is smooth and $\mathcal{L}$ is very ample, the homomorphism $j_{1, x}$ has rank $n+1$ at every point $x \in X$. We are interested in the generic rank of the homomorphism $j_{k}$ for $k \geq 2$. Choose local coordinates $\left(u_{1}, \ldots, u_{m}, v_{m+1}, \ldots, v_{n}\right)$ around $x \in X$ in such a way that $\left(u_{1}, \ldots, u_{m}\right)$ are (pullbacks of) local coordinates around $\pi(x)$ on $Y$, while $\left(v_{m+1}, \ldots, v_{n}\right)$ are local coordinates around $x$ on the fiber $\pi^{-1}(\pi(x)) \cong \mathbb{P}^{n-m}$. Then, any section $\sigma \in V$ can be represented, locally around $x$, in the following form:

$$
\sigma=a\left(u_{1}, \ldots, u_{m}\right)+\sum_{j=m+1}^{n} b_{j}\left(u_{1}, \ldots, u_{m}\right) v_{j} .
$$

Since $\sigma$ is linear in the variables $v_{j}$ for $j=m+1, \ldots, n$, when we take the derivatives of order $h$ for $2 \leq h \leq k$, only the following are not obviously zero: $\sigma_{u_{1}^{i_{1}}, \ldots, u_{m}^{i m}}$ with $\sum_{j=1}^{m} i_{j}=h$ and $\sigma_{u_{1}^{i_{1}}, \ldots, u_{m}^{i m}, v_{j}}$ with $\sum_{j=1}^{m} i_{j}=h-1$ and $j=$ $m+1, \ldots n$. Those of the former type are counted by the forms of degree $h$ in $\left(u_{1}, \ldots, u_{m}\right)$, and hence their number is $\left(\begin{array}{c}m-1+h \\ h\end{array}\right)$; those of the latter type, once $v_{j}$ is fixed, are counted by the forms of degree $h-1$ in $\left(u_{1}, \ldots, u_{m}\right)$, which are $\left(\begin{array}{c}m-1+h-1 \\ h-1\end{array}\right)$, and hence their total number is $(n-m)\left(\begin{array}{c}m-1+h-1 \\ h-1\end{array}\right)$. Let $s_{k}$ be the generic rank of $j_{k}$. Then the above says that $s_{k}$ cannot exceed

$$
n+1+\sum_{h=2}^{k}\left(\left(\begin{array}{c}
m-1+h \\
h
\end{array}\right)+(n-m)\left(\begin{array}{c}
m-1+h-1 \\
h-1
\end{array}\right)\right) .
$$

Lemma 2.1. The generic rank $s_{k}$ of $j_{k}$ satisfies

$$
s_{k} \leq r_{k},
$$

where

$$
r_{k}:=(n-m)\left(\begin{array}{c}
m+k-1 \\
k-1
\end{array}\right)+\left(\begin{array}{c}
m+k \\
k
\end{array}\right) .
$$

Proof. Use the fact that

$$
\sum_{h=0}^{k}\left(\begin{array}{c}
m-1+h \\
h
\end{array}\right)=\left(\begin{array}{c}
m+k \\
k
\end{array}\right)
$$


Example 2.2. Let $Y \subset \mathbb{P}^{M}$ be an $m$-dimensional smooth variety, let $\mathcal{H}$ be the hyperplane line bundle, and consider the scroll $X:=\mathbb{P}_{Y}\left(\mathcal{H}^{\oplus(n-m+1)}\right) \subset \mathbb{P}^{N}$, where $N=(M+1)(n-m+1)-1$. Clearly, $X \cong Y \times \mathbb{P}^{n-m}$, embedded by the Segre embedding of $\mathbb{P}^{M} \times \mathbb{P}^{n-m} \rightarrow \mathbb{P}^{N}$. Fix any point $x \in X$ and let $y:=\pi(x)$, where $\pi: X \rightarrow Y$ is the projection. Arguing as in [15, p. 1049-1050] or [8, Lemma 2.1], one can express the matrix representing $j_{k, x}$ for $X \subset \mathbb{P}^{N}$ in terms of the blocks representing $j_{k, y}$ and $j_{k-1, y}$ for $Y \subset \mathbb{P}^{M}$, showing that

$$
\mathrm{rk} j_{k, x}=(n-m) \mathrm{rk} j_{k-1, y}+\mathrm{rk} j_{k, y} .
$$

In particular, we see that in this special case, equality holds in (2.1) if and only if the base variety $Y \subset \mathbb{P}^{M}$ is generically $k$-regular. Moreover, recalling that $\Phi_{k-1}(Y) \subseteq \Phi_{k}(Y)$, we see that $\Phi_{k}(X)=\pi^{-1}\left(\Phi_{k}(Y)\right)$. So, either $X$ is uninflected, i.e., $\Phi_{k}(X)=\varnothing$, or $\operatorname{dim} \Phi_{k}(X)=\operatorname{dim} \Phi_{k}(Y)+(n-m)$. In particular, $X$ is uninflected if and only if $Y$ is.

Example 2.3. Here are some examples of scrolls for which (2.1) is a strict inequality. First of all, we mention the Segre products (a) $X=\mathbb{P}^{2} \times \mathbb{P}^{1} \subset \mathbb{P}^{5}$ and (b) $X=\mathbb{P}^{1} \times \mathbb{P}^{1} \times \mathbb{P}^{1} \subset \mathbb{P}^{7}$. According to what we said in Example 2.2, they cannot satisfy the equality $s_{2}=r_{2}$. This also follows from the fact that $r_{2}=9$, while the dimension $N$ of the embedding space is too small in both cases. Note that in the former case, $X$ is also a scroll over $\mathbb{P}^{1}$, so this situation is covered by [9]. Look at the latter case: Since $X=\mathbb{P}_{\mathbb{P}^{1} \times \mathbb{P}^{1}}\left(\mathcal{O}(1,1)^{\oplus 2}\right)$, it follows from Example 2.2 that $\mathrm{rk} j_{2, x}=7$ for every $x \in X$. This makes $X$ an example of a perfectly hypo-osculating threefold scroll, but, unfortunately, we cannot include it in our subsequent discussion.

More generally, we can consider the following Segre products:

(A) $X=\mathbb{P}^{m} \times \mathbb{P}^{n-m} \subset \mathbb{P}^{N}$, where $N=m(n-m)+n$. By using coordinates $\left(1: u_{1}: \cdots: u_{m}\right)$ on $\mathbb{P}^{m}$ and $\left(1: v_{1}: \cdots: v_{n-m}\right)$ on $\mathbb{P}^{n-m}, X$ is locally represented in $\mathbb{P}^{N}$ by coordinates

$$
\left(1: \cdots: u_{i}: \cdots: v_{j}: \cdots: u_{i} v_{j}: \ldots\right) .
$$

Clearly all second derivatives of such coordinates are zero, and we immediately see that $j_{2, x}$ has rank $1+m+(n-m)+m(n-m)$ at every point $x \in X$. Thus $s_{2}=N+1$. On the other hand, from (2.2) we get $r_{2}=(n-m)(m+1)+\left(\begin{array}{c}m+2 \\ 2\end{array}\right)$. Therefore $s_{2}=r_{2}-\left(\begin{array}{c}m+1 \\ 2\end{array}\right)$.

(B) $X=\left(\mathbb{P}^{1}\right)^{n} \subset \mathbb{P}^{2^{n}-1}$. Clearly, $X=\mathbb{P}_{Y}\left(\mathcal{O}_{Y}(1,1, \ldots, 1)^{\oplus 2}\right)$, where $Y=$ $\left(\mathbb{P}^{1}\right)^{n-1}$. Thus, if $u_{i}$ is a local parameter on the $i$ th factor of $Y, i=$ $1, \ldots, n-1$, then $X$ is locally represented in $\mathbb{P}^{2^{n}-1}$ by coordinates

$$
\left(1: u_{1}: \cdots: v: u_{1} u_{2}: \cdots: u_{n-1} v: \cdots: u_{1} \ldots u_{n-1} v\right) .
$$

Since any such coordinate $\sigma$ is linear in the parameters, we have that in the matrix representing $j_{2, x}$ the $n-1$ rows representing the second derivatives 
$\sigma_{u_{j}^{2}}$ for $j=1, \ldots, n-1$ (in addition to $\sigma_{v^{2}}$ ) are zero. The remaining rows are clearly linearly independent; hence $s_{2}=1+n+\left(\begin{array}{c}n \\ 2\end{array}\right)=\left(\begin{array}{c}n+2 \\ 2\end{array}\right)-n=$ $r_{2}-(n-1)$. In fact, it is easy to see that

$$
s_{n-1}=1+\left(\begin{array}{c}
n \\
1
\end{array}\right)+\cdots+\left(\begin{array}{c}
n \\
n-1
\end{array}\right)=2^{n}-1<r_{n-1}=3\left(\begin{array}{c}
2 n-3 \\
n-1
\end{array}\right)
$$

and that $X$ is perfectly hypo-osculating.

Another interesting threefold scroll excluded from our discussion, that we would like to mention, is $\mathbb{P}\left(T_{\mathbb{P}^{2}}\right) \subset \mathbb{P}^{7}$, for which we have $s_{2}=8<9=r_{2}$.

Next, let $Y\left(\cong \mathbb{F}_{1}\right) \subset \mathbb{P}^{4}$ be the rational cubic surface scroll, and let $X=$ $Y \times \mathbb{P}^{1} \subset \mathbb{P}^{9}$, embedded via the Segre embedding. As $Y$ is locally described by ( $1: u_{1}: u_{2}: u_{1} u_{2}: u_{1}^{2} u_{2}$ ), $X$ is locally represented by

$$
\left(1: u_{1}: u_{2}: v: u_{1} u_{2}: u_{1} v: u_{2} v: u_{1} u_{2} v: u_{1}^{2} u_{2}: u_{1}^{2} u_{2} v\right)
$$

Note that no term of degree $\geq 2$ in $u_{2}$ appears. Hence $s_{2} \leq 8$, and in fact a direct check shows that $s_{2}=8$. Thus $s_{2}<r_{2}=9$.

We want to investigate the inflectional loci of nondegenerate, smooth scrolls over a smooth base space. We shall do this under the assumption that equality holds in (2.1). Note that this is a serious restriction, as the previous examples show.

Theorem 2.4. Let $X \subset \mathbb{P}^{N}$ be an n-dimensional scroll over a smooth $m$ dimensional variety $Y$, with hyperplane bundle $\mathcal{L}=\left.\mathcal{O}_{P^{N}}(1)\right|_{X}$. For given $k \geq 1$, set $r_{k}=(n-m)\left(\begin{array}{c}m+k-1 \\ m\end{array}\right)+\left(\begin{array}{c}m+k \\ m\end{array}\right)$. Assume that the generic rank of $j_{k}$ is equal to $r_{k}$ for all $k \geq 1$ such that $r_{k} \leq N+1$. Set $Q_{k}=\operatorname{Coker} j_{k}$. Then, for all such $k$,

(i) $\mathcal{Q}_{k}^{\vee}$ is a locally free sheaf of rank $\left(\begin{array}{c}n+k \\ n\end{array}\right)-r_{k}$;

(ii) there exist locally free sheaves $\mathcal{M}_{k}$, of rank $\left(\begin{array}{c}n+k-1 \\ n-1\end{array}\right)-\left(\begin{array}{c}m+k-1 \\ m-1\end{array}\right)-(n-m)\left(\begin{array}{c}m+k-2 \\ m-1\end{array}\right)$, and $\mathcal{T}_{k}$, of rank $(n-m)\left(\begin{array}{c}m+k-2 \\ m-1\end{array}\right)+\left(\begin{array}{c}m+k-1 \\ m-1\end{array}\right)$, and exact sequences

$$
0 \rightarrow \mathcal{Q}_{k-1}^{\vee} \rightarrow \mathcal{Q}_{k}^{\vee} \rightarrow \mathcal{M}_{k}^{\vee} \rightarrow 0
$$

and

$$
0 \rightarrow \mathcal{T}_{k} \rightarrow S^{k} \Omega_{X} \otimes L \rightarrow \mathcal{M}_{k} \rightarrow 0
$$

(iii) the quotient sheaves $\mathcal{E}_{k}:=\mathcal{P}_{X}^{k}(\mathcal{L})^{\vee} / \mathcal{Q}_{k}^{\vee}$ are locally free, of rank $r_{k}$, and there exist exact sequences

$$
0 \rightarrow \mathcal{E}_{k-1} \rightarrow \mathcal{E}_{k} \rightarrow \mathcal{T}_{k}^{\vee} \rightarrow 0
$$


Proof. We will adapt the proof of [9, Theorem 1]. Define the sheaf $\mathcal{E}_{k}$ by the exact sequence

$$
0 \rightarrow \mathcal{Q}_{k}^{\vee} \rightarrow \mathcal{P}_{X}^{k}(\mathcal{L})^{\vee} \rightarrow \mathcal{E}_{k} \rightarrow 0 .
$$

Set $\mathcal{K}_{k}:=\operatorname{ker}\left(S^{k-1} \Omega_{Y} \otimes \Omega_{Y} \rightarrow S^{k} \Omega_{Y}\right)$; then we have an exact sequence

$$
0 \rightarrow \mathcal{K}_{k} \rightarrow S^{k-1} \Omega_{Y} \otimes \Omega_{Y} \rightarrow S^{k} \Omega_{Y} \rightarrow 0,
$$

and $\mathcal{K}_{k}$ is locally free, with rank $m\left(\begin{array}{c}m+k-2 \\ m-1\end{array}\right)-\left(\begin{array}{c}m+k-1 \\ m-1\end{array}\right)$. We observe that $\pi^{*} \mathcal{K}_{k}$ is the kernel of the composite map

$$
\pi^{*} S^{k-1} \Omega_{Y} \otimes \Omega_{X} \rightarrow S^{k-1} \Omega_{X} \otimes \Omega_{X} \rightarrow S^{k} \Omega_{X} .
$$

To see this, let $x \in X$, let $u_{1}, \ldots, u_{m}$ denote local coordinates on the base variety $Y$ around $\pi(x)$, and let $v_{m+1}, \ldots, v_{n}$ denote local coordinates on the fiber through $x$ around $x$. So, if $A:=\mathcal{O}_{X, x}$, we have the following isomorphisms:

$$
\begin{aligned}
\left(\pi^{*} \Omega_{Y}\right)_{x} & \cong A \mathrm{~d} u_{1} \oplus \cdots \oplus A \mathrm{~d} u_{m}, \\
\Omega_{X, x} & \cong \bigoplus_{i=1}^{m} A \mathrm{~d} u_{i} \oplus \bigoplus_{j=m+1}^{n} A \mathrm{~d} v_{j}, \\
\left(S^{k-1} \Omega_{X}\right)_{x} & \cong \bigoplus_{i_{1}, \ldots, i_{n}} A \mathrm{~d} u_{1}^{i_{1}} \cdots \mathrm{d} u_{m}^{i_{m}} \mathrm{~d} v_{m+1}^{i_{m+1}} \cdots \mathrm{d} v_{n}^{i_{n}},
\end{aligned}
$$

with $\sum_{j} i_{j}=k-1$. The composite map (2.4) can be described locally in this way:

$$
d u_{1}^{i_{1}} \cdots \mathrm{d} u_{m}^{i_{m}} \otimes \mathrm{d} u_{j} \mapsto \mathrm{d} u_{1}^{i_{1}} \cdots \mathrm{d} u_{j}^{i_{j}+1} \cdots \mathrm{d} u_{m}^{i_{m}},
$$

for $j=1, \ldots, m$, and

$$
d u_{1}^{i_{1}} \cdots \mathrm{d} \boldsymbol{u}_{m}^{i_{m}} \otimes \mathrm{d} \boldsymbol{v}_{h} \mapsto \mathrm{d} \boldsymbol{u}_{1}^{i_{1}} \cdots \mathrm{d} \boldsymbol{u}_{m}^{i_{m}} \mathrm{~d} \boldsymbol{v}_{h},
$$

for $h=m+1, \ldots, n$.

The kernel is generated by the elements

$$
\begin{aligned}
\mathrm{d} \boldsymbol{u}_{1}^{i_{1}} \cdots \mathrm{d} \boldsymbol{u}_{j}^{i_{j}} \cdots \mathrm{d} \boldsymbol{u}_{\ell}^{i_{\ell}-1} \cdots \mathrm{d} \boldsymbol{u}_{m}^{i_{m}} \otimes \mathrm{d} \boldsymbol{u}_{\ell} & \\
& -\mathrm{d} \boldsymbol{u}_{1}^{i_{1}} \cdots \mathrm{d} \boldsymbol{u}_{j}^{i_{j}-1} \cdots \mathrm{d} \boldsymbol{u}_{\ell}^{i_{\ell}} \cdots \mathrm{d} \boldsymbol{u}_{m}^{i_{m}} \otimes \mathrm{d} \boldsymbol{u}_{j},
\end{aligned}
$$

where $i_{1}+\cdots+i_{m}=k, j \neq \ell$, and $i_{j}, i_{\ell} \geq 1$. These generators are not linearly independent when $m>2$. As $\pi^{*} \mathcal{K}_{k}$ is a subbundle of $\pi^{*} S^{k-1} \Omega_{Y} \otimes \Omega_{X}$ via the map

$$
0 \rightarrow \pi^{*} S^{k-1} \Omega_{Y} \otimes \pi^{*} \Omega_{Y} \rightarrow \pi^{*} S^{k-1} \Omega_{Y} \otimes \Omega_{X},
$$


it is obvious that it coincides with the kernel just computed.

Moreover, the composite map (2.4) has constant rank, so its image is a subbundle of $S^{k} \Omega_{X}$. Local computations show that the image in $S^{k} \Omega_{X}$ is generated by $\left\{\mathrm{d} u_{1}^{i_{1}} \cdots \mathrm{d} \boldsymbol{u}_{m}^{i_{m}} ; \mathrm{d} u_{1}^{i_{1}^{\prime}} \cdots \mathrm{d} \boldsymbol{u}_{m}^{i_{m}^{\prime}} \mathrm{d} \boldsymbol{v}_{h}\right\}$ with $\sum i_{j}=k, \sum i_{j}^{\prime}=k-1, h=$ $m+1, \ldots, n$. These generators are independent, and their number is $\left(\begin{array}{c}m+k-1 \\ m-1\end{array}\right)+$ $(n-m)\left(\begin{array}{c}m+k-2 \\ m-1\end{array}\right)$. This is valid for all points $x \in X$. Tensoring with $\mathcal{L}$, we thus get an exact sequence

$$
0 \rightarrow \pi^{*} \mathcal{K}_{k} \otimes \mathcal{L} \rightarrow \pi^{*} S^{k-1} \Omega_{Y} \otimes \Omega_{X} \otimes \mathcal{L} \rightarrow \mathcal{T}_{k} \rightarrow 0,
$$

where

$$
\mathcal{T}_{k}:=\frac{\pi^{*} S^{k-1} \Omega_{Y} \otimes \Omega_{X} \otimes \mathcal{L}}{\pi^{*} \mathcal{K}_{k} \otimes \mathcal{L}}
$$

is a subbundle of $S^{k} \Omega_{X} \otimes \mathcal{L}$. By arguing as in [9, pp. 559-560], this allows us to obtain the following exact diagram:

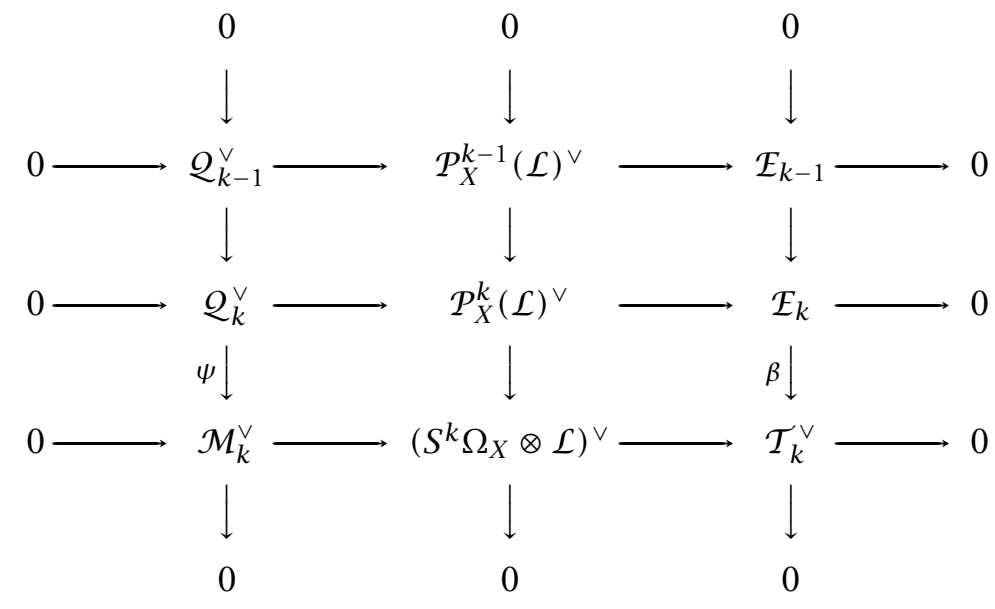

In particular, the third vertical sequence above,

$$
0 \rightarrow \mathcal{E}_{k-1} \rightarrow \mathcal{E}_{k} \rightarrow \mathcal{T}_{k}^{\vee} \rightarrow 0
$$

shows by induction on $k$ that $\mathcal{E}_{k}$ is a vector bundle (note that $\mathcal{E}_{0}=\mathcal{L}^{-1}$ and $\left.\mathcal{E}_{1}=\mathcal{P}_{X}^{1}(\mathcal{L})^{\vee}\right)$.

Theorem 2.5. Let $X \subset \mathbb{P}^{N}$ be an $n$-dimensional scroll over a smooth $m$ dimensional variety $Y$, with hyperplane bundle $\mathcal{L}=\left.\mathcal{O}_{\mathbb{P}^{N}}(1)\right|_{X}$. Let $\pi: X \rightarrow Y$ denote the projection, and set $\mathcal{V}:=\pi_{*} \mathcal{L}$. Set $r_{k}=(n-m)\left(\begin{array}{c}m+k-1 \\ m\end{array}\right)+\left(\begin{array}{c}m+k \\ m\end{array}\right)$ for given $k \geq 1$. Assume that the generic rank of $j_{k}$ is equal to $r_{k}$ for all $k \geq 1$ such 
that $r_{k} \leq N+1$. Assume that the kth inflectional locus $\Phi_{k}$ of $X$ has codimension $\ell:=N+2-r_{k}$ or is empty. Then $\Phi_{k}$ has a natural structure as a Cohen-Macaulay scheme, and its class is given as

$$
\left[\Phi_{k}\right]=\left[\prod_{i=0}^{k-1} \pi^{*} c\left(S^{i} T_{Y} \otimes \mathcal{V}^{\vee}\right)^{-1} c\left(\pi^{*} S^{k} T_{Y} \otimes \mathcal{L}^{-1}\right)^{-1}\right]_{\ell} .
$$

Proof. The $k$ th inflectional locus of $X$, i.e., the locus $\Phi_{k}:=\Phi_{k}(X)$ where $j_{k}: V_{X} \rightarrow \mathcal{P}_{X}^{k}(\mathcal{L})$ drops rank, is the same as the locus where the map $j_{k}^{\vee}: \mathcal{E}_{k} \rightarrow V_{X}^{\vee}$ drops rank. Locally the map is given by an $(N+1) \times r_{k}$ matrix. Hence the expected codimension of $\Phi_{k}$ is $\ell:=N+1-\left(r_{k}-1\right)$. Then the first part of the statement in the theorem and the fact that $\left[\Phi_{k}\right]=\left[c\left(\mathcal{E}_{k}\right)^{-1}\right]_{\ell}$ follow from Porteous's formula [5, Example 14, p. 255] if we observe that $c\left(V_{X}^{\vee}\right) c\left(\mathcal{E}_{k}\right)^{-1}=c\left(\mathcal{E}_{k}\right)^{-1}$.

To make this expression explicit, we then compute the total Chern class $c\left(\mathcal{E}_{k}\right)$ by recursion. Since $\mathcal{E}_{0}=\mathcal{L}^{-1}$, we get

$$
c\left(\mathcal{E}_{k}\right)=\prod_{i=1}^{k} c\left(\mathcal{T}_{i}^{\vee}\right) c\left(\mathcal{L}^{-1}\right) .
$$

To compute the total Chern class of $\mathcal{T}_{i}^{\vee}$, dualizing (2.5), we get

$$
0 \rightarrow \mathcal{T}_{i}^{\vee} \rightarrow \pi^{*} S^{i-1} T_{Y}^{i} \otimes T_{X} \otimes \mathcal{L}^{-1} \rightarrow \pi^{*} \mathcal{K}_{i}^{\vee} \otimes \mathcal{L}^{-1} \rightarrow 0,
$$

where $T_{Y}$ and $T_{X}$ are the tangent bundles of $Y$ and $X$. Hence

$$
c\left(\mathcal{T}_{i}^{\vee}\right)=c\left(\pi^{*} S^{i-1} T_{Y}^{i} \otimes T_{X} \otimes \mathcal{L}^{-1}\right) c\left(\pi^{*} \mathcal{K}_{i}^{\vee} \otimes \mathcal{L}^{-1}\right)^{-1} .
$$

Note that $X=\mathbb{P}(\mathcal{V})$, since $\mathcal{V}=\pi_{*} \mathcal{L}$, so that $\mathcal{L}$ is the tautological line bundle on $X$. Recall the relative tangent and Euler exact sequences

$$
\begin{aligned}
& 0 \rightarrow T_{X / Y} \rightarrow T_{X} \rightarrow \pi^{*} T_{Y} \rightarrow 0, \\
& 0 \rightarrow \mathcal{O}_{X} \rightarrow \pi^{*} \mathcal{V}^{\vee} \otimes \mathcal{L} \rightarrow T_{X / Y} \rightarrow 0 .
\end{aligned}
$$

Now, twisting (2.8) by $\pi^{*} S^{i-1} T_{Y} \otimes \mathcal{L}^{-1}$, we get the exact sequence:

$$
\begin{aligned}
0 & \rightarrow \pi^{*} S^{i-1} T_{Y} \otimes T_{X / Y} \otimes \mathcal{L}^{-1} \rightarrow \pi^{*} S^{i-1} T_{Y} \otimes T_{X} \otimes \mathcal{L}^{-1} \\
& \rightarrow \pi^{*}\left(S^{i-1} T_{Y} \otimes T_{Y}\right) \otimes \mathcal{L}^{-1} \rightarrow 0 .
\end{aligned}
$$

On the other hand, twisting by $\mathcal{L}^{-1}$ the dual of (2.3) lifted to $X$ via $\pi^{*}$, we obtain a second exact sequence:

$$
0 \rightarrow \pi^{*} S^{i} T_{Y} \otimes \mathcal{L}^{-1} \rightarrow \pi^{*}\left(S^{i-1} T_{Y} \otimes T_{Y}\right) \otimes \mathcal{L}^{-1} \rightarrow \pi^{*} \mathcal{K}_{i}^{\vee} \otimes \mathcal{L}^{-1} \rightarrow 0 .
$$


Using both, we can rewrite (2.7) as

$$
c\left(\mathcal{T}_{i}^{\vee}\right)=c\left(\pi^{*} S^{i-1} T_{Y} \otimes T_{X / Y} \otimes \mathcal{L}^{-1}\right) c\left(\pi^{*} S^{i} T_{Y} \otimes \mathcal{L}^{-1}\right) .
$$

Now, twisting (2.9) by $\pi^{*} S^{i-1} T_{Y} \otimes \mathcal{L}^{-1}$, we can replace the first total Chern class appearing on the right-hand side of the above equality, obtaining

$$
c\left(\mathcal{T}_{i}^{\vee}\right)=\pi^{*} c\left(S^{i-1} T_{Y} \otimes \mathcal{V}^{\vee}\right) c\left(\pi^{*} S^{i-1} T_{Y} \otimes \mathcal{L}^{-1}\right)^{-1} c\left(\pi^{*} S^{i} T_{Y} \otimes \mathcal{L}^{-1}\right) .
$$

Note that the last two terms cancel in the product $\prod_{i=1}^{k} c\left(\mathcal{T}_{i}^{\vee}\right)$, except the second for $i=1$, which is $c\left(\mathcal{L}^{-1}\right)^{-1}$, and the third for $i=k$. Thus, because of these cancellations, (2.6) gives

$$
c\left(\mathcal{E}_{k}\right)=\prod_{i=1}^{k} \pi^{*} c\left(S^{i-1} T_{Y} \otimes \mathcal{V}^{\vee}\right) c\left(\pi^{*} S^{k} T_{Y} \otimes \mathcal{L}^{-1}\right),
$$

which shows what we wanted.

Note that we can also write, using Segre classes,

$$
c\left(\mathcal{F}_{k}\right)^{-1}=s\left(\mathcal{F}_{k}^{\vee}\right)=\prod_{i=1}^{k} \pi^{*} s\left(S^{i-1} \Omega_{Y} \otimes \mathcal{V}\right) s\left(\pi^{*} S^{k} \Omega_{Y} \otimes \mathcal{L}\right) .
$$

As to the assumptions in Theorem 2.5, note that the requirement that $\Phi_{k}$ have the expected codimension $\ell$ is independent of the condition $s_{k}=r_{k}$, as the following example shows.

Example 2.6. Let $\mathcal{V}$ be a vector bundle of rank 2 on $\mathbb{P}^{2}$ fitting into an exact sequence

$$
0 \rightarrow \mathcal{O}_{\mathbb{P} 2}(1)^{\oplus 2} \rightarrow T_{\mathbb{P} 2}^{\oplus 2} \rightarrow \mathcal{V} \rightarrow 0 .
$$

Then $\mathcal{V}$ is very ample and $c_{1}(\mathcal{V})=4, c_{2}(\mathcal{V})=6$. If we let $X:=\mathbb{P}(\mathcal{V})$, the tautological line bundle $\mathcal{L}$ embeds $X$ in $\mathbb{P}^{9}$ as a scroll of degree 10. In fact, $X$ can be regarded as a general threefold section of $\mathbb{P}^{2} \times \mathbb{P}^{3} \subset \mathbb{P}^{11}$, embedded via the Segre embedding, with a linear space [10, Section 3]. Sometimes one refers to $X$ as a Bordiga scroll, since its general hyperplane section $S \in|\mathcal{L}|$ is a Bordiga surface (namely, a rational surface of sectional genus 3 ). According to [11, Theorem 3], $X$ is also isomorphic to $\mathbb{P}^{3}$ blown-up along a twisted cubic $C$ and, by [10, Lemma 1.4], $\mathcal{L}=\sigma^{*} \mathcal{O}_{\mathbb{P}^{3}}(3) \otimes \mathcal{O}_{X}(-E)$, where $\sigma$ is the blowing-up and $E$ is the exceptional divisor. Thus $V=H^{0}(X, \mathcal{L}) \cong H^{0}\left(\mathbb{P}^{3}, J_{C}(3)\right)$, where $\mathcal{J}_{C}$ is the ideal sheaf of $C \subset \mathbb{P}^{3}$. We study $\Phi_{2}(X)$ relying on this description. Using coordinates $(1: x: y: z)$ on $\mathbb{P}^{3}$, let $C$ be defined by $\left(1: t: t^{2}: t^{3}\right)$. Consider the three quadrics $Q_{1}, Q_{2}, Q_{3}$ containing $C$, defined by $x^{2}-y, x y-z$, $x z-y^{2}$, respectively. Taking into account the syzygies $x Q_{2}=y Q_{1}-Q_{3}$ and 
$z Q_{1}=-y Q_{2}+x Q_{3}$, we can easily find a basis for $H^{0}\left(\mathbb{P}^{3}, \mathcal{J}_{C}(3)\right)$. Moreover, as $\sigma$ is an isomorphism outside $E$, we can use the affine coordinates $(x, y, z)$ around any point of $\mathbb{P}^{3} \backslash C$ as local coordinates around the corresponding point $p \in X \backslash E$. Then $X \backslash E$ can be represented in $\mathbb{P}^{9}$, near $p$, by the following homogeneous coordinates:

$$
\left(Q_{1}: x Q_{1}: y Q_{1}: Q_{2}: y Q_{2}: z Q_{2}: Q_{3}: x Q_{3}: y Q_{3}: z Q_{3}\right)
$$

Now computing the rank of $j_{2, p}$ (e. g., by using Maple ${ }^{\mathrm{TM}}$ [12]), we obtain that $s_{2}=9=r_{2}$. Actually, computing the minors of order 9 , one can easily find that the locus they define is $y=x^{2}, z=x^{3}$. This means that $\mathrm{rk} j_{2, p}=9$ for every $p \in X \backslash E$. Hence $\Phi_{2} \subseteq E$. To look at $E$, note that the plane $\Pi \subset \mathbb{P}^{3}$ defined by $x=0$ is transverse to $C$ at the point $o=(0,0,0)$. If we take $(y, w)$ as local coordinates on $\tilde{\Pi}:=\sigma^{-1}(\Pi)$, the local equations of $\sigma$ restricted to $\tilde{\Pi}$ are $\sigma(0, y, w)=(0, y, z=y w)$. Therefore the homogeneous coordinates in $\mathbb{P}^{9}$ of points $p \in X$, near the fiber $f_{o} \subset E$ of $\left.\sigma\right|_{E}$, are given by the polynomials $x^{2}-y, x^{3}-x y, x^{2} y-y^{2}, x y-y w, x y^{2}-y^{2} w, x y^{2} w-y^{2} w^{2}, x y w-y^{2}$, $x^{2} y w-x y^{2}, x y^{2} w-y^{3}, x y^{2} w^{2}-y^{3} w$, in terms of the local parameters $x$, $y, w$ (one simply has to replace $z$ with $y w$ in the previous polynomials). Now redoing the computation (with Maple ${ }^{\mathrm{TM}}[12]$ ), we see that the rank of $j_{2, p}$ is 9 , for $p$ in a neighborhood $U$ of $f_{o}$ in $X$. But all $9 \times 9$-minors contain the factor $y$ with some multiplicity. Since $y=0$ is the local equation of $E$ in $U$, this means that $\left(\Phi_{2}\right)_{\text {red }}=E$. In particular, $\Phi_{2}$ is a divisor. We conclude that $s_{2}=r_{2}$, but $\Phi_{2}$ does not have the expected codimension $\ell=2$.

The assumption that the inflectional locus has codimension $\ell$ implies that $\ell$ is in the range $1 \leq \ell \leq n$, which means

$$
1 \leq N+2-r_{k} \leq n \text {. }
$$

This implies that $k$ is the maximal integer such that $r_{k} \leq N+1$ holds, as we will now explain. First of all, $\ell \geq 1$ is equivalent to $r_{k} \leq N+1$, and $\ell \leq n$ is equivalent to $r_{k} \geq N+2-n$. If this holds, then since

$$
r_{k+1}=r_{k}+\left(\begin{array}{c}
m+k \\
m-1
\end{array}\right)+(n-m)\left(\begin{array}{c}
m-1+k \\
m-1
\end{array}\right),
$$

we get

$$
r_{k+1} \geq N+2-n+\left(\begin{array}{c}
m+k \\
m-1
\end{array}\right)+(n-m)\left(\begin{array}{c}
m-1+k \\
m-1
\end{array}\right) .
$$

Hence it suffices to show that

$$
-n+\left(\begin{array}{c}
m+k \\
m-1
\end{array}\right)+(n-m)\left(\begin{array}{c}
m-1+k \\
m-1
\end{array}\right) \geq 0 .
$$


The left-hand side can be written as

$$
n\left(\left(\begin{array}{c}
m-1+k \\
m-1
\end{array}\right)-1\right)-k\left(\begin{array}{c}
m-1+k \\
m-2
\end{array}\right)
$$

which is the same as

$$
k\left((n-1)+\left(\frac{n}{2}-1\right)(k+1)+\cdots+\left(\frac{n}{n-m}-1\right)\left(\begin{array}{c}
m-2+k \\
m-2
\end{array}\right)\right),
$$

and this is clearly nonnegative. Hence $r_{k+1} \geq N+2$.

If $Y$ is a curve (see [9]), so $m=1$, then $r_{k}=k n+1$, and in this case, if $k$ is the largest integer such that $r_{k} \leq N+1$, then conversely $1 \leq \ell \leq n$ holds. When $m \geq 2$, this last implication is no longer true. However, if we take $k$ to be the largest integer such that $r_{k-1}-1+n \leq N$, then the bound on $\ell \leq n$ holds (recall that $r_{k-1}-1$ is the dimension of the $(k-1)$ th osculating space to $X$ at a general point). To see this, assume $r_{k}-1+n \geq N+1$. Then $r_{k} \geq N+2-n$, so that

$$
\ell=N+2-r_{k} \leq N+2-N-2+n=n .
$$

The scrolls for which Theorem 2.5 applies are thus those such that the general $k$ th osculating spaces are of dimension $r_{k}-1$ and where $r_{k}$ satisfies

$$
r_{k}-1 \leq N \leq r_{k}+n-2 .
$$

For instance, the appropriate range for studying $\Phi_{2}$ is

$$
(n-m)(m+1)+\left(\begin{array}{c}
m+2 \\
2
\end{array}\right)-1 \leq N \leq(n-m)(m+1)+\left(\begin{array}{c}
m+2 \\
2
\end{array}\right)+n-2 .
$$

For $m=2$ this gives

$$
3 n-1 \leq N \leq 4 n-2,
$$

and for $m=3$,

$$
4 n-3 \leq N \leq 5 n-4 .
$$

In the special cases $m=2, n=3$ and $m=3, n=4$ that we will consider in Sections 6 and 7, this means that $8 \leq N \leq 10$ and $13 \leq N \leq 16$, respectively.

Note, however, that (2.11) in no way implies that $s_{k}=r_{k}$. For instance, for $\left(\mathbb{P}^{1}\right)^{4} \subset \mathbb{P}^{15}$ condition (2.12) is satisfied, but, as observed in Example 2.3, we have $s_{2}=11<14=r_{2}$.

Sometimes in the following we say that $X \subset \mathbb{P}^{N}$ satisfies our general assumptions to mean that $s_{k}=r_{k}$ and $\Phi_{k}$ has the expected codimension. From Section 4 on, we in addition assume $k=2$. 


\section{The Case $Y$ Is an Abelian Variety}

In this section we assume that $X \subset \mathbb{P}^{N}$ is a scroll over an abelian variety $Y$. We use the same notation as in the previous section: $\pi: X \rightarrow Y$ is the scroll projection, $\mathcal{L}$ is the hyperplane bundle on $X$, and $\mathcal{V}=\pi_{*} \mathcal{L}$.

In this case, we have $T_{Y}=\mathcal{O}_{Y}^{\oplus m}$, so

$$
S^{j} T_{Y}=\mathcal{O}_{Y}^{\oplus\left(\begin{array}{c}
m-1+j \\
m-1
\end{array}\right)} \text { and } \quad \pi^{*} S^{j} T_{Y}=\mathcal{O}_{X}^{\oplus\left(\begin{array}{c}
m-1+j \\
m-1
\end{array}\right)} .
$$

Set $\mu:=\left(\begin{array}{c}m-1+k \\ m-1\end{array}\right)$. Then

$$
c\left(\pi^{*} S^{k} T_{Y} \otimes \mathcal{L}^{-1}\right)=c\left(\pi^{*} \mathcal{O}_{Y}^{\oplus \mu} \otimes \mathcal{L}^{-1}\right)=c\left(\left(\mathcal{L}^{-1}\right)^{\oplus \mu}\right)=(1-L)^{\mu},
$$

where we set $L:=c_{1}(\mathcal{L})$. Similarly,

$$
\prod_{i=0}^{k-1} c\left(S^{i} T_{Y} \otimes \mathcal{V}^{\vee}\right)=\prod_{i=0}^{k-1} c\left(\mathcal{V}^{\vee}\right)^{\left(\begin{array}{c}
m-1+i \\
m-1
\end{array}\right)}=c\left(\mathcal{V}^{\vee}\right)^{v},
$$

where $v:=\sum_{i=0}^{k-1}\left(\begin{array}{c}m-1+i \\ m-1\end{array}\right)=\left(\begin{array}{c}m-1+k \\ m\end{array}\right)$. Therefore we get from (2.10)

$$
c\left(\mathcal{E}_{k}\right)=(1-L)^{\mu} \pi^{*} c\left(\mathcal{V}^{\vee}\right)^{v} .
$$

Now we have to compute the inverse

$$
c\left(\mathcal{E}_{k}\right)^{-1}=(1-L)^{-\mu} \pi^{*} c\left(\mathcal{V}^{\vee}\right)^{-\nu} .
$$

By the general binomial formula,

$$
(1-L)^{-\mu}=\sum_{j=0}^{\infty}\left(\begin{array}{c}
j+\mu-1 \\
\mu-1
\end{array}\right) L^{j} .
$$

Set $V_{i}=\pi^{*} c_{i}(\mathcal{V})$. We have

$$
\begin{aligned}
\pi^{*} c\left(\mathcal{V}^{\vee}\right)^{-v} & =\left(1-V_{1}+V_{2}-V_{3}+\cdots\right)^{-v} \\
& =\sum_{h=0}^{\infty}\left(\begin{array}{c}
h+v-1 \\
v-1
\end{array}\right)\left(V_{1}-V_{2}+\cdots+(-1)^{m+1} V_{m}\right)^{h}
\end{aligned}
$$

All this gives

$$
\begin{aligned}
c\left(\mathcal{E}_{k}\right)^{-1}= & \sum_{j=0}^{n}\left(\begin{array}{c}
j+\mu-1 \\
\mu-1
\end{array}\right) L^{j} \\
& \times \sum_{h=0}^{m}\left(\begin{array}{c}
h+v-1 \\
v-1
\end{array}\right)\left(V_{1}-V_{2}+\cdots+(-1)^{m+1} V_{m}\right)^{h} .
\end{aligned}
$$


Now let us put $m=2$. Then we get the following result.

Proposition 3.1. Let $X \subset \mathbb{P}^{N}$ be a $n$-dimensional scroll over an abelian surface $Y$, with projection $\pi: X \rightarrow Y$, and let $\mathcal{V}=\pi_{*} \mathcal{L}$, where $\mathcal{L}$ is the hyperplane bundle. Suppose that (2.11) is satisfied, that $j_{k}$ has maximal general rank, and that the inflectional locus $\Phi_{k}$ has the expected codimension $\ell$ or is empty. Then the cohomology class of $\Phi_{k}$ is

$$
\begin{aligned}
{\left[\Phi_{k}\right]=} & \left(\begin{array}{c}
\ell+k \\
k
\end{array}\right) L^{\ell}+v\left(\begin{array}{c}
\ell-1+k \\
k
\end{array}\right) V_{1} L^{\ell-1} \\
& +\left(\begin{array}{c}
\ell-2+k \\
k
\end{array}\right)\left[\left(\begin{array}{c}
v+1 \\
2
\end{array}\right) V_{1}^{2}-v V_{2}\right] L^{\ell-2},
\end{aligned}
$$

where $L=c_{1}(\mathcal{L})$ and $V_{i}=\pi^{*} c_{i}(\mathcal{V})$. In particular,

$$
\begin{aligned}
\operatorname{deg} \Phi_{k}= & {\left[\left(\begin{array}{c}
\ell+k \\
k
\end{array}\right)+\left(\begin{array}{c}
k+1 \\
2
\end{array}\right)\left(\begin{array}{c}
\ell-2+k \\
k
\end{array}\right)\right] d } \\
& +\left[\left(\begin{array}{c}
k+1 \\
2
\end{array}\right)\left(\begin{array}{c}
\ell-1+k \\
k
\end{array}\right)+\left(\begin{array}{c}
k+1 \\
2 \\
2
\end{array}\right)\left(\begin{array}{c}
\ell-2+k \\
k
\end{array}\right)\right](2 g-2),
\end{aligned}
$$

where $d$ and $g$ are the degree and the sectional genus of $X$.

Proof. The formula for $\left[\Phi_{k}\right]$ follows from Theorem 2.5 and (3.1). To get the degree it is enough to dot with $L^{n-\ell}$ and to recall the following facts: $d=L^{n}=$ $L^{n-2}\left(V_{1}^{2}-V_{2}\right)$ and $L^{n-1} \pi^{*} D=c_{1}(\mathcal{V}) D$ for any divisor $D$ on $Y$, by the ChernWu relation. Moreover, for the general surface section $S$ of $X$, the pair $(Y, \operatorname{det} \mathcal{V})$ is the adjunction theoretic reduction of $\left(S, \mathcal{L}_{S}\right)$ via the map $\left.\pi\right|_{S}: S \rightarrow Y$. Then $g=g(X, \mathcal{L})=g\left(S, \mathcal{L}_{S}\right)=g(Y, \operatorname{det} \mathcal{V})$; hence $c_{1}(\mathcal{V})^{2}=2 g-2$ by the genus formula.

In particular, suppose that $\Phi_{k}$ has codimension $\ell=n$, which implies $N=$ $n(1+v)-1-\left(\begin{array}{l}k \\ 2\end{array}\right)$. Then $X$ has finitely many flexes, their number being given by

$$
\begin{aligned}
\operatorname{deg} \Phi_{k}= & {\left[\left(\begin{array}{c}
n+k \\
k
\end{array}\right)+\left(\begin{array}{c}
k+1 \\
2
\end{array}\right)\left(\begin{array}{c}
n-2+k \\
k
\end{array}\right)\right] d } \\
& +\left[\left(\begin{array}{c}
k+1 \\
2
\end{array}\right)\left(\begin{array}{c}
n-1+k \\
k
\end{array}\right)+\left(\begin{array}{c}
k+1 \\
2 \\
2
\end{array}\right)\left(\begin{array}{c}
n-2+k \\
k
\end{array}\right)\right](2 g-2) .
\end{aligned}
$$

For instance, for $\ell=n=3$ and $k=2$, we get $\operatorname{deg} \Phi_{2}=19 d+27(2 g-2)$. Note that $d$ and $g$ are large; actually, $\operatorname{det} \mathcal{V}$ is a very ample line bundle on $Y$, since $\mathcal{V}=\pi_{*} \mathcal{L}$ is a very ample vector bundle. So, in a sense, the above formula shows that any $n$-dimensional scroll $X$ over an abelian surface is very, very far from being uninflected. 
Example 3.2. Let $A$ be an abelian surface with Picard number 1, and let $\mathcal{H}$ be a line bundle on $A$ representing a polarization of type $(1, p)$ with $p=(k+1)^{2}+2$, $k \geq 2$. Then $\mathcal{H}$ is very ample by Reider's theorem. Moreover, $H^{0}(A, \mathcal{H})$ embeds $A$ in $\mathbb{P}^{N}$ with $N=p-1$ as a surface of degree $2 p$. Let $\varepsilon \in A$ be a nontrivial element of order 2 , let $Y$ be the quotient $A / \varepsilon$, and let $q: A \rightarrow Y$ be the quotient map, which is an isogeny of degree 2 . Set $\mathcal{V}:=q_{*} \mathcal{H}$. Then $\mathcal{V}$ is a rank-2 vector bundle on $Y$. As $q^{*} \mathcal{V}=\mathcal{H} \oplus t_{\varepsilon}^{*} \mathcal{H}$, where $t_{\varepsilon}: A \rightarrow A$ is the translation by $\varepsilon$ [3, (1), p. 360], we get

$$
\left(c_{1}(\mathcal{V})^{2}, c_{2}(\mathcal{V})\right)=(4 p, p)
$$

by the functoriality of the Chern classes. Now let $X:=\mathbb{P}(\mathcal{V})$ and let $\mathcal{L}$ be the tautological line bundle. Note that $p>9$. Then [3, Theorem 3.3] implies the following facts: $\mathcal{L}$ is very ample, $H^{0}(X, \mathcal{L})$ embeds $X$ in $\mathbb{P}^{N}$, and if we identify this space with that containing $A$ via the isomorphisms

$$
H^{0}(A, \mathcal{H}) \cong H^{0}(Y, \mathcal{V}) \cong H^{0}(X, \mathcal{L}),
$$

the image is the 3-dimensional scroll over $Y$ generated by the secant lines joining $x$ and $x+\varepsilon$, as $x$ varies on $A$. Note that its degree is $d=c_{1}(\mathcal{V})^{2}-c_{2}(\mathcal{V})=$ $3 p$. Moreover, condition (2.11) is satisfied with equality on the right, because $r_{k}+n-2=\left(\begin{array}{c}k+1 \\ 2\end{array}\right)+\left(\begin{array}{c}k+2 \\ 2\end{array}\right)+1=(k+1)^{2}+1=N$. Thus for this abelian scroll, our formula allows us to express $\operatorname{deg} \Phi_{k}$ as a degree 5 polynomial in $k$, provided that $\Phi_{k}$ is a finite set. The explicit expression is the following:

$$
\operatorname{deg} \Phi_{k}=\frac{p}{2}\left(k^{5}+5 k^{4}+13 k^{3}+19 k^{2}+16 k+6\right) .
$$

In particular, for $k=2$ we get $\operatorname{deg} \Phi_{2}=1815$.

This example can be generalized to scrolls of any dimension $n \geq 3$ over an abelian surface by taking $p=n\left(\left(\begin{array}{c}k+1 \\ 2\end{array}\right)+1\right)-\left(\begin{array}{l}k \\ 2\end{array}\right)$ and $Y:=A / G$, where $G \subset A$ is the subgroup generated by a nontrivial element $\rho$ of order $n-1$. In this case $\mathcal{V}=$ $q_{*} \mathcal{H}$ is a vector bundle of rank $n-1$, and $q^{*} \mathcal{V}=\mathcal{H} \oplus t_{\rho}^{*} \mathcal{H} \oplus \cdots \oplus t_{(n-2) \rho}^{*} \mathcal{H}$. As before, let $X:=\mathbb{P}(\mathcal{V})$, so that $X$ is an $n$-dimensional scroll over $Y$, and let $\mathcal{L}$ be the tautological line bundle again. Since $A$ has Picard number 1 and the inequality $\mathcal{H}^{2} \geq 4(2 n-1)+6$ is satisfied for every $n \geq 3$ and $k \geq 2$, we know from [22, Theorem 1.1] that $\mathcal{H}$ is $(2 n-1)$-very ample on $A$. Then [4, Proposition 2.2] tells us that $\mathcal{L}$ is very ample, $H^{0}(X, \mathcal{L})$ embeds $X$ in $\mathbb{P}^{N}$, and if we identify this space with that containing $A$ as before, the image is the $n$-dimensional scroll over $Y$ generated by the $(n-2)$-dimensional linear spaces $\langle x, x+\rho, \ldots, x+(n-1) \rho\rangle$, as $x$ varies on $A$. Condition (2.11) is satisfied and $\ell=n$ again. Then our formula for $\operatorname{deg} \Phi_{k}$ applies also in this case, provided that $\Phi_{k}$ is a finite set.

For $m=3$ we get the following result. 
Proposition 3.3. Let $X \subset \mathbb{P}^{N}$ be an $n$-dimensional scroll over an abelian threefold $Y$, with projection $\pi: X \rightarrow Y$, and let $\mathcal{V}=\pi_{*} \mathcal{L}$, where $\mathcal{L}$ is the hyperplane bundle. Suppose that (2.11) is satisfied, that $j_{k}$ has maximal general rank, and that the inflectional locus $\Phi_{k}$ has the expected codimension $\ell$. Set $L=c_{1}(\mathcal{L})$ and $V_{i}=\pi^{*} c_{i}(\mathcal{V})$. Then the cohomology class of $\Phi_{k}$ is

$$
\begin{aligned}
{\left[\Phi_{k}\right]=} & \left(\begin{array}{c}
\ell+\mu-1 \\
\mu-1
\end{array}\right) L^{\ell}+v\left(\begin{array}{c}
\ell+\mu-2 \\
\mu-1
\end{array}\right) V_{1} L^{\ell-1} \\
& +\left(\begin{array}{c}
\ell+\mu-3 \\
\mu-1
\end{array}\right)\left(\left(\begin{array}{c}
v+1 \\
2
\end{array}\right) V_{1}^{2}-v V_{2}\right) L^{\ell-2} \\
& +\left(\begin{array}{c}
\ell+\mu-4 \\
\mu-1
\end{array}\right)\left(\left(\begin{array}{c}
v+2 \\
3
\end{array}\right) V_{1}^{3}-v(v+1) V_{1} V_{2}+v V_{3}\right) L^{\ell-3},
\end{aligned}
$$

where $\mu=\left(\begin{array}{c}k+2 \\ 2\end{array}\right)$ and $v=\left(\begin{array}{c}k+2 \\ 3\end{array}\right)$.

\section{The CASe $k=2$ : When $\Phi_{2}$ Is A Divisor}

From now on we consider the case $k=2$. In this section we study the inflectional locus $\Phi_{2}$, assuming it is a divisor. First of all, we prove the following result:

Theorem 4.1. Let $X \subset \mathbb{P}^{N}$ be an $n$-dimensional scroll over a smooth $m$ dimensional variety $Y$ with projection $\pi$, and let $\mathcal{V}=\pi_{*} \mathcal{L}$, where $\mathcal{L}$ is the hyperplane bundle. Suppose that the conditions on the range of $N$ are satisfied, that $j_{2}$ has maximal general rank, and that the inflectional locus $\Phi_{2}$ is a divisor. Then its class is given by

$$
\left[\Phi_{2}\right]=\pi^{*}\left((n+2) K_{Y}+(m+1) c_{1}(\mathcal{V})\right)+\left(\begin{array}{c}
m+1 \\
2
\end{array}\right) L,
$$

where $L=c_{1}(\mathcal{L})$.

Proof. Letting $k=2$, we reduce (2.10) to the product of three terms

$$
c\left(\mathcal{E}_{2}\right)=\pi^{*} c\left(\mathcal{V}^{\vee}\right) \pi^{*} c\left(\mathcal{V}^{\vee} \otimes T_{Y}\right) c\left(\pi^{*} S^{2} T_{Y} \otimes \mathcal{L}^{-1}\right) .
$$

According to Theorem 2.5, to determine the class of $\Phi_{2}$ we have to take the inverse of the expression in (4.1). If $\Phi_{2}$ is a divisor, i.e., $\ell=1$, we need only the degree 1 term. In other words,

$$
\left[\Phi_{2}\right]=\alpha_{1}+\beta_{1}+\gamma_{1},
$$

where $\alpha_{1}, \beta_{1}$, and $\gamma_{1}$ are the terms of degree 1 appearing in the expressions of $c\left(\pi^{*} \mathcal{V}^{\vee}\right)^{-1}, c\left(\pi^{*} \mathcal{V}^{\vee} \otimes T_{Y}\right)^{-1}$, and $c\left(\pi^{*} S^{2} T_{Y} \otimes \mathcal{L}^{-1}\right)^{-1}$, respectively. Thus

$$
\alpha_{1}=-c_{1}\left(\pi^{*} \mathcal{V}^{\vee}\right)=\pi^{*} c_{1}(\mathcal{V}) \text {. }
$$


Similarly, $\beta_{1}=-c_{1}\left(\pi^{*} \mathcal{V}^{\vee} \otimes T_{Y}\right)$. By confining the use of the splitting principle to $\mathcal{V}$, we can easily see that

$$
\beta_{1}=(n-m+1) \pi^{*} K_{Y}+m \pi^{*} c_{1}(\mathcal{V}) .
$$

Finally, $\gamma_{1}=-c_{1}\left(\pi^{*} S^{2} T_{Y} \otimes \mathcal{L}^{-1}\right)$. Noting that the rank of $S^{2} T_{Y}$ is $\left(\begin{array}{c}m+1 \\ 2\end{array}\right)$ and $c_{1}\left(S^{2} T_{Y}\right)=(m+1) c_{1}\left(T_{Y}\right)$, we get

$$
\gamma_{1}=(m+1) \pi^{*} K_{Y}+\left(\begin{array}{c}
m+1 \\
2
\end{array}\right) L .
$$

Adding the three terms, we thus get the expression in the statement.

Example 4.2. Consider the scroll over $\mathbb{P}^{m}$ given by $X=\mathbb{P}(\mathcal{V})$, where $\mathcal{V}=$ $\mathcal{O}_{\mathbb{P} m}(1) \oplus \mathcal{O}_{\mathbb{P} m}(2)$. Here $n=m+1$, and the tautological line bundle $\mathcal{L}$ embeds $X \subset \mathbb{P}^{N}$, where $N=\left(\begin{array}{c}m+2 \\ m\end{array}\right)+m$. Note that, according to (2.12), we are in the appropriate range for studying $\Phi_{2}$ with $\ell=1$, so that $\Phi_{2}$ is a divisor. Let $Y_{0} \subset X$ be the section corresponding to the summand $\mathcal{O}_{\mathbb{P} m}(1)$. Equivalently, we can look at $Y_{0}$ as the tautological section of the vector bundle $\mathcal{V} \otimes \mathcal{O}_{\mathbb{P} m}(-2)$; hence $Y_{0}$ is negative and $L=Y_{0}+2 \pi^{*} \mathcal{O}_{\mathbb{p} m}(1)$. By applying Theorem 4.1 we thus get

$$
\Phi_{2}(X)=\left(\begin{array}{c}
m+1 \\
2
\end{array}\right) Y_{0}
$$

This allows us to point out another relevant discrepancy with respect to the case of scrolls over curves. Let $m=1$. Then our $X$ is the cubic surface scroll $X \subset \mathbb{P}^{4}$, and $Y_{0}$ is its directrix line. Recall that $r_{2}=5$ for $m=1$. Parameterizing $X$, locally around $Y_{0}$, by

$$
\left(1: u: v: v u: v u^{2}\right),
$$

we see that the inflectional locus $\Phi_{2}$ is defined by $v=0$; hence it consists of $Y_{0}$, whose codimension is the expected one, and $\mathrm{rk} j_{2, x}=4$ for every $x \in \Phi_{2}$, i.e., one less than the generic rank. By applying [9, Corollary 1] to this case, we get $\left[\Phi_{2}\right]=L-2 F$, where $L$ and $F$ are the classes of a hyperplane and a fiber, respectively. And this agrees with the fact that $Y_{0} \in|L-2 F|$. Now let $m=2$ and note that here $r_{2}=9$. If we parameterize $X$, locally around $Y_{0}$, by

$$
\left(1: u_{1}: u_{2}: v: v u_{1}: v u_{2}: v u_{1}^{2}: v u_{1} u_{2}: v u_{2}^{2}\right),
$$

a direct check shows that $s_{2}=9$ and $\Phi_{2}$ is defined by $v^{3}=0$. So $\Phi_{2}$ is nonreduced. In fact, $\Phi_{2}(X)=3 Y_{0}$, according to the above formula. Set-theoretically, $\Phi_{2}=Y_{0}$ and is of the expected codimension, but $\mathrm{rk} j_{2, x}=6=r_{2}-3$ for every $x \in \Phi_{2}$. In other words, the rank of $j_{2}$ drops by 3 on the inflectional locus. From the geometric point of view, this fact can be rephrased as follows. Take any 
point $x \in Y_{0}$, let $\left\{\lambda_{t}\right\}$ be the pencil of lines in $Y_{0}$ passing through $x$, and set $S_{t}=\mathbb{P}\left(\mathcal{V}_{\lambda_{t}}\right)$. Then $\operatorname{Osc}_{x}^{2}(X)=\left\langle\bigcup_{t} \operatorname{Osc}_{x}^{2}\left(S_{t}\right)\right\rangle$. Note that, for every $t$, $\operatorname{Osc}_{x}^{2}\left(S_{t}\right)$ is a $\mathbb{P}^{3}$ containing the fiber of $X$ through $x$. This says that $\operatorname{Osc}_{x}^{2}(X)$ is a $\mathbb{P}^{5}$. Moreover, we see that $Y_{0}$, which is the union of the directrix lines constituting the $\Phi_{2}\left(S_{t}\right)$ as $\lambda_{t}$ varies, is equal to $\Phi_{2}(X)_{\text {red }}$.

Remark 4.3. Suppose $\Phi_{2}$ is a divisor. Theorem 4.1 implies that

$$
\left(\Phi_{2}\right)_{f} \in\left|\mathcal{O}_{\mathbb{P} n-m}\left(\left(\begin{array}{c}
m+1 \\
2
\end{array}\right)\right)\right|
$$

for any fiber $f$ of $X$. This prevents $\Phi_{2}$ from being empty, since by definition $\Phi_{2}$ is either effective or empty. Therefore $X$ is not uninflected. Moreover, since $f$ is a linear $\mathbb{P}^{n-m}$, for every line $\lambda \subset f$ the condition $\Phi_{2} \lambda=\left(\begin{array}{c}m+1 \\ 2\end{array}\right)$ implies that

$$
\operatorname{deg} \Phi_{2} \geq\left(\begin{array}{c}
m+1 \\
2
\end{array}\right)
$$

Otherwise, every fiber $f$ would be contained in $\Phi_{2}$, implying that $\Phi_{2}(X)=X$, a contradiction. Note that for the scroll considered in the above example, we have in fact $\operatorname{deg} \Phi_{2}=\left(\begin{array}{c}m+1 \\ 2\end{array}\right)$.

Dotting the expression of $\Phi_{2}$ provided by Theorem 4.1 with $L^{n-1}$, we get

$$
\operatorname{deg} \Phi_{2}=\pi^{*}\left((n+2) K_{Y}+(m+1) c_{1}(\mathcal{V})\right) L^{n-1}+\left(\begin{array}{c}
m+1 \\
2
\end{array}\right) d,
$$

where $d$ is the degree of $X$. For instance, let $m=2$, so that $\mathcal{V}$ has rank $n-1$. As we observed in the proof of Proposition 3.1, the sectional genus of $X$ is $g=$ $g(Y, \operatorname{det} \mathcal{V})$. Then, taking into account the Chern-Wu relation and genus formula, we get the following two equivalent expressions for the degree:

$$
\begin{aligned}
\operatorname{deg} \Phi_{2} & =(4-n) d+(n+2)(2 g-2)-(n-1) c_{2}(\mathcal{V}) \\
& =(4-n) c_{1}(\mathcal{V})^{2}+(n+2)(2 g-2)-3 c_{2}(\mathcal{V}) .
\end{aligned}
$$

From (4.2) we immediately get the following consequence.

Corollary 4.4. Let $X, \mathcal{L}, Y$, and $\mathcal{V}$ be as in Theorem 4.1. If the $\mathbb{Q}$-divisor $K_{Y}+(m+1) /(n+2) \operatorname{det} \mathcal{V}$ is nef, then

$$
\operatorname{deg} \Phi_{2} \geq\left(\begin{array}{c}
m+1 \\
2
\end{array}\right) d
$$

where $d$ is the degree of $X$. 
Note that $(m+1) /(n+2) \leq(m+1) /(m+3)=1-2 /(m+3)$. Hence the adjunction bundle appearing in the statement does not fit with the range of the nef values of adjoint bundles to an ample vector bundle considered by Ohno in [13]. This prevents us from giving a more detailed statement in the general case. However, restricting to threefold scrolls over surfaces, we can produce a precise result in the same vein as Corollary 4.4. This will be done in the next section.

\section{The Divisor $\Phi_{2}$ FOr Threefold Scrolls OVER Surfaces}

Let $X=\mathbb{P}(\mathcal{V})$ be a threefold scroll over a smooth surface $Y$, satisfying our general assumptions and with $\ell=1$, which implies that $X \subset \mathbb{P}^{8}$. The expression provided by Theorem 4.1 becomes

$$
\left[\Phi_{2}\right]=\pi^{*}\left(5 K_{Y}+3 c_{1}(\mathcal{V})\right)+3 L .
$$

We start by recalling the following well-known facts (e.g., for (A2), see [6, Proposition 5.2]).

Remark 5.1. Let $\mathcal{V}$ be an ample vector bundle of rank 2 on a smooth projective variety $Y$.

(A1) If $C \subset Y$ is a smooth rational curve, then $\operatorname{deg} \mathcal{V}_{C} \geq 2$, and equality implies that $\mathcal{V}_{C} \cong \mathcal{O}_{\mathbb{P} 1}(1)^{\oplus 2}$; moreover, if $\operatorname{deg} \mathcal{V}_{C}=3$, then $\mathcal{V}_{C} \cong \mathcal{O}_{\mathbb{P} 1}(2) \oplus$ $\mathcal{O}_{\mathbb{P} 1}(1)$.

(A2) Suppose that $\mathcal{V}$ is very ample, and let $C \subset Y$ be a smooth curve of genus 1 ; then $\operatorname{deg} \mathcal{V}_{C} \geq 5$.

Now, let $Y$ be a smooth surface, and let $\mathcal{V}$ be a very ample vector bundle of rank 2 on $Y$. We want to investigate the effectiveness of $5 K_{Y}+3 \operatorname{det} \mathcal{V}$. First of all, we consider the adjoint line bundle $A:=K_{Y}+\operatorname{det} \mathcal{V}$. As usual in adjunction theory, we use the additive notation for the tensor product of line bundles on $Y$.

Proposition 5.2. The line bundle $A$ is very ample except in the following cases:

(1) $(Y, \mathcal{V})=\left(\mathbb{P}^{2}, \mathcal{O}_{\mathbb{P}^{2}}(1)^{\oplus 2}\right)$;

(2) $(Y, \mathcal{V})=\left(\mathbb{P}^{2}, \mathcal{O}_{\mathbb{P}^{2}}(2) \oplus \mathcal{O}_{\mathbb{P}^{2}}(1)\right)$;

(3) $(Y, \mathcal{V})=\left(\mathbb{P}^{2}, T_{\mathbb{P}^{2}}\right)$, where $T_{\mathbb{P}^{2}}$ is the tangent bundle;

(4) $(Y, \mathcal{V})=\left(\mathbb{P}^{1} \times \mathbb{P}^{1}, \mathcal{O}_{\mathbb{P}^{1} \times \mathbb{P}^{1}}(1,1)^{\oplus 2}\right)$;

(5) $Y$ is a $\mathbb{P}^{1}$-bundle over a smooth curve $B$, and $\mathcal{V}_{f}=\mathcal{O}_{\mathbb{P}^{1}}(1)^{\oplus 2}$ for every fiber $f$ of the bundle projection $p: Y \rightarrow B$.

Proof. Consider the pair $(Y, \operatorname{det} \mathcal{V})$. According to [20, Theorem 0.1] and taking into account Remark 5.1 (A1), $A$ is nef unless $(Y, \operatorname{det} \mathcal{V})=\left(\mathbb{P}^{2}, \mathcal{O}_{\mathbb{P}^{2}}(2)\right)$. This gives exception (1), due to Remark 5.1 (A1) again and the uniformity of $\mathcal{V}$. Now, according to [20, Theorem 0.2], $A$ is big unless $Y$ is a Del Pezzo surface with $\operatorname{det} \mathcal{V}=-K_{Y}$ or $(Y, \operatorname{det} \mathcal{V})$ is a conic fibration over a smooth curve $B$. In the former case, $Y$ has to be a minimal surface in view of Remark 5.1 (A1); hence $(Y, \operatorname{det} \mathcal{V})$ is either $\left(\mathbb{P}^{2}, \mathcal{O}_{\mathbb{P}^{2}}(3)\right)$ or $\left(\mathbb{P}^{1} \times \mathbb{P}^{1}, \mathcal{O}_{\mathbb{P}^{1} \times \mathbb{P}^{1}}(2,2)\right)$. This gives exceptions (2), (3), and (4), due to Remark 5.1 (A1) again and the uniformity of $\mathcal{V}$ (e.g., see 
[14, Theorem 2.2.2] and [23, Lemma 3.6.1]). If $(Y, \operatorname{det} \mathcal{V})$ is a conic fibration, then all fibers of the projection $p: Y \rightarrow B$ are irreducible by Remark 5.1 (A1); hence $Y$ is in fact a $\mathbb{P}^{1}$-bundle over $B$, and we get case (5), by Remark 5.1 (A1) again. So, apart from cases (1)-(5), $A$ is nef and big, and then we can consider the adjunction theoretic reduction of $(Y, \operatorname{det} \mathcal{V})$ [20, Theorem 0.3$]$, which coincides with $(Y, \operatorname{det} \mathcal{V})$ itself, once more in view of Remark 5.1 (A1). Then, according to [20, Theorem 2.1 and Theorem 1.5] and Remark 5.1 (A1), we have that $A$ is very ample, except in the following cases:

(i) $Y$ is a Del Pezzo surface with $K_{Y}^{2}=1$ and $\operatorname{det} \mathcal{V}=-3 K_{Y}$;

(ii) $Y$ is a Del Pezzo surface with $K_{Y}^{2}=2$ and $\operatorname{det} \mathcal{V}=-2 K_{Y}$;

(iii) $Y$ is an elliptic $\mathbb{P}^{1}$-bundle of invariant -1 with $\operatorname{det} \mathcal{V}$ linearly equivalent to $3 \sigma$, where $\sigma$ is a section of minimal self-intersection.

However, they cannot occur in our setting. Actually, in all these cases $Y$ contains a smooth curve $C$ of genus 1 such that $\operatorname{deg} \mathcal{V}_{C}<5$ (take $C \in\left|-K_{Y}\right|$ in cases (i), (ii) and $C=\sigma$ in case (iii)). This contradicts Remark 5.1 (A2).

Proposition 5.3. Suppose that the adjoint line bundle $A$ is very ample. Then $h^{0}\left(K_{Y}+A\right)>0$ except in the following cases:

(1) $(Y, \operatorname{det} \mathcal{V})=\left(\mathbb{P}^{2}, \mathcal{O}_{\mathbb{P}^{2}}(4)\right)$;

(2) $(Y, \operatorname{det} \mathcal{V})=\left(\mathbb{P}^{2}, \mathcal{O}_{\mathbb{P}^{2}}(5)\right)$;

(3) $Y$ is a $\mathbb{P}^{1}$-bundle over a smooth curve $B$, and $\mathcal{V}_{f}=\mathcal{O}_{\mathbb{P}^{1}}(2) \oplus \mathcal{O}_{\mathbb{P}^{1}}(1)$ for every fiber $f$ of the bundle projection $p: Y \rightarrow B$.

Proof. Let $C \in|A|$ be a smooth curve, and let $g^{\prime}:=g(C)$. From the cohomology sequence of

$$
0 \rightarrow K_{Y} \rightarrow K_{Y}+A \rightarrow\left(K_{Y}+A\right)_{C} \rightarrow 0,
$$

recalling that $h^{0}\left(\left(K_{Y}+A\right)_{C}\right)=h^{0}\left(K_{C}\right)=g^{\prime}$, we immediately get, in view of the Kodaira vanishing theorem,

$$
h^{0}\left(K_{Y}+A\right)=p_{g}(Y)+g^{\prime}-q(Y) .
$$

Note that $g^{\prime} \geq q(Y)$ by the Lefschetz theorem, with equality if and only if ( $\left.Y, A\right)$ is either $\left(\mathbb{P}^{2}, \mathcal{O}_{\mathbb{P}^{2}}(a)\right)$, with $a=1,2$, or a scroll over a smooth curve $B$ [19, Corollary 1.5.2]. The former case leads to (1) and (2) respectively, while in the latter case, we have $\operatorname{deg} \mathcal{V}_{f}=3$ for every fiber $f$ of the projection $p: Y \rightarrow B$, since $K_{Y} f=-2$. Then, taking into account Remark 5.1 (A1), we get (3). We thus see that $h^{0}\left(K_{Y}+A\right) \geq 1$ apart from (1)-(3).

Corollary 5.4. Suppose that $(Y, \mathcal{V})$ is not any of the exceptions listed in Proposition 5.2 and Proposition 5.3. Then the linear system $\left|5 K_{Y}+3 \operatorname{det} \mathcal{V}\right|$ contains an effective nontrivial divisor. 
Proof. By our assumptions, we know that $A$ is very ample and $h^{0}\left(K_{Y}+A\right) \geq 1$. Let $C \in|A|$ be a smooth curve. Tensoring the exact sequence

$$
0 \rightarrow-A \rightarrow \mathcal{O}_{Y} \rightarrow \mathcal{O}_{C} \rightarrow 0
$$

by $2\left(K_{Y}+A\right)+A$, we get the exact sequence

$$
0 \rightarrow 2 K_{Y}+2 A \rightarrow 5 K_{Y}+3 \operatorname{det} \mathcal{V} \rightarrow\left(5 K_{Y}+3 \operatorname{det} \mathcal{V}\right)_{C} \rightarrow 0
$$

Therefore

$$
h^{0}\left(5 K_{Y}+3 \operatorname{det} \mathcal{V}\right) \geq h^{0}\left(2\left(K_{Y}+A\right)\right) \geq h^{0}\left(K_{Y}+A\right) \geq 1 .
$$

Suppose $5 K_{Y}+3 \operatorname{det} \mathcal{V}$ is trivial. Then $Y$ is a Del Pezzo surface, and $-K_{Y}$ is divisible by 3 in the Picard group. This means that $Y=\mathbb{P}^{2}$ and $\operatorname{det} \mathcal{V}=\frac{5}{3}\left(-K_{Y}\right)=$ $\mathcal{O}_{\mathbb{P}^{2}}(5)$. But then $(Y, \mathcal{V})$ would be as in case (2) of Proposition 5.3, a contradiction. Therefore $\left|5 K_{Y}+3 \operatorname{det} \mathcal{V}\right|$ contains an effective nontrivial divisor.

Let us analyze more closely the various exceptions that have arisen in the discussion. In cases (1)-(4) of Proposition 5.2, clearly $5 K_{Y}+3 \operatorname{det} \mathcal{V}$ is not effective. The same is true for case (1) of Proposition 5.3, while, as already observed, $5 K_{Y}+3 \operatorname{det} \mathcal{V}$ is trivial in case (2) of Proposition 5.3. To complete the discussion, it remains to consider case (5) of Proposition 5.2 and case (3) of Proposition 5.3. In both cases, for every fiber $f$ of the projection $p: Y \rightarrow B$, we know that $K_{Y} f=-2$ and $\operatorname{deg} \mathcal{V}_{f}=2$ or 3 , respectively. Hence $\left(5 K_{Y}+3 \operatorname{det} \mathcal{V}\right) f<0$ in both cases. Therefore $5 K_{Y}+3 \operatorname{det} \mathcal{V}$ is not effective. Let us add something more on these two cases. First of all, we can write $Y=\mathbb{P}(\mathcal{F})$ for an ample vector bundle $\mathcal{F}$ of $\operatorname{rank} 2$ on $B$. Then $K_{Y}=-2 \xi+p^{*}\left(K_{B}+\operatorname{det} \mathcal{F}\right)$, where $\xi$ is the tautological line bundle on $Y$.

In case (5) of Proposition 5.2, since $\mathcal{V} \otimes \xi^{-1}$ restricts trivially to every fiber $f$ of $p$, there exists a vector bundle $G$ of rank 2 on $B$ such that $\mathcal{V}=\xi \otimes p^{*} G$. Thus $\operatorname{det} \mathcal{V}=2 \xi+p^{*} \operatorname{det} \mathcal{G}$ and $c_{2}(\mathcal{V})=\xi^{2}+\xi p^{*} \operatorname{det} \mathcal{G}=\operatorname{deg} \mathcal{F}+\operatorname{deg} \mathcal{G}$ by the Chern-Wu relation. Thus

$$
0<c_{1}(\mathcal{V})^{2}=4(\operatorname{deg} \mathcal{F}+\operatorname{deg} \mathcal{G})=4 c_{2}(\mathcal{V}) .
$$

In case (3) of Proposition 5.3 we have $(\mathcal{V} \otimes[-2 \xi])_{f}=\mathcal{O}_{\mathbb{P}^{1}} \oplus \mathcal{O}_{\mathbb{P}^{1}}(-1)$ for every fiber $f$ of $p$. Hence $p_{*}(\mathcal{V} \otimes[-2 \xi])$ is a line bundle, say, $\mathcal{A} \in \operatorname{Pic}(B)$, and we have an obvious injection giving rise to an exact sequence

$$
0 \rightarrow 2 \xi+p^{*} \mathcal{A} \rightarrow \mathcal{V} \rightarrow \mathcal{Q} \rightarrow 0,
$$

where $\mathcal{Q}$ is a very ample line bundle on $Y$, since it is a quotient of $\mathcal{V}$. This gives $\operatorname{det} \mathcal{V}=2 \xi+p^{*} \mathcal{A}+\mathcal{Q}$, and from the relation $3=\operatorname{deg} \mathcal{V}_{f}=\left(2 \xi+p^{*} \mathcal{A}+\mathcal{Q}\right) f=$ $2+\mathcal{Q} f$, we conclude that $\mathcal{Q}=\xi+p^{*} \mathcal{M}$ for some line bundle $\mathcal{M} \in \operatorname{Pic}(B)$. Thus $\operatorname{det} \mathcal{V}=3 \xi+p^{*}(\mathcal{A}+\mathcal{M})$. Note also that

$$
c_{2}(\mathcal{V})=2 \xi^{2}+\xi p^{*}(\mathcal{A}+2 \mathcal{M})=2 \operatorname{deg} \mathcal{F}+\operatorname{deg} \mathcal{A}+2 \operatorname{deg} \mathcal{M}
$$


by the Chern-Wu relation. Thus

$$
\begin{aligned}
c_{1}(\mathcal{V})^{2} & =9 \operatorname{deg} \mathcal{F}+6(\operatorname{deg} \mathcal{A}+\operatorname{deg} \mathcal{M}) \\
& =c_{2}(\mathcal{V})+(7 \operatorname{deg} \mathcal{F}+5 \operatorname{deg} \mathcal{A}+4 \operatorname{deg} \mathcal{M}) .
\end{aligned}
$$

The previous discussion allows us to provide a significant lower bound for $\operatorname{deg} \Phi_{2}$ of threefold scrolls in $\mathbb{P}^{8}$, apart from very restricted cases.

Theorem 5.5. Let $X \subset \mathbb{P}^{8}$ be a threefold scroll of degree d over a smooth surface $Y$, with projection $\pi$, and let $\mathcal{V}=\pi_{*} \mathcal{L}$, where $\mathcal{L}$ is the hyperplane line bundle. Suppose that $X$ satisfies our general assumptions. Then $\operatorname{deg} \Phi_{2} \geq 3 d$ unless

(1) $(Y, \mathcal{V})=\left(\mathbb{P}^{2}, \mathcal{O}_{\mathbb{P}^{2}}(2) \oplus \mathcal{O}_{\mathbb{P}^{2}}(1)\right)$, where $\left(d, \operatorname{deg} \Phi_{2}\right)=(7,3)$;

(2) $(Y, \operatorname{det} \mathcal{V})=\left(\mathbb{P}^{2}, \mathcal{O}_{\mathbb{P}^{2}}(4)\right)$, in which case $\operatorname{deg} \Phi_{2}=3 d-12$;

(3) $Y=\mathbb{P}(\mathcal{F})$ for some ample vector bundle $\mathcal{F}$ of rank 2 over a smooth curve $B$ of genus $\mathcal{q}$, and $\mathcal{V}=\xi \otimes p^{*} \mathcal{G}$, where $\xi$ is the tautological line bundle, $p: Y \rightarrow B$ is the bundle projection, and $G$ is a vector bundle of rank 2 on $B$, in which case

$$
\operatorname{deg} \Phi_{2}=3 d+20(q-1)+2(\operatorname{deg} \mathcal{F}+\operatorname{deg} \mathcal{G})
$$

(4) $Y=\mathbb{P}(\mathcal{F})$ for some ample vector bundle $\mathcal{F}$ of rank 2 over a smooth curve $B$ of genus $q$, and $\mathcal{V}$ fits into an exact sequence

$$
0 \rightarrow 2 \xi+p^{*} \mathcal{A} \rightarrow \mathcal{V} \rightarrow \xi+p^{*} \mathcal{M} \rightarrow 0,
$$

where $\xi$ is the tautological line bundle, $p: Y \rightarrow B$ is the bundle projection, and $\mathcal{A}$ and $\mathcal{M}$ are line bundles on $B$, in which case

$$
\operatorname{deg} \Phi_{2}=3 d+30(q-1)+12 \operatorname{deg} \mathcal{F}+8(\operatorname{deg} \mathcal{A}+\operatorname{deg} \mathcal{M}) .
$$

Moreover, equality $\operatorname{deg} \Phi_{2}=3 d$ holds if and only if $(Y, \operatorname{det} \mathcal{V})=\left(\mathbb{P}^{2}, \mathcal{O}_{\mathbb{P}^{2}}(5)\right)$.

Proof. Dotting formula (5.1) with $L^{2}$ and taking into account Corollary 5.4, we see that $\operatorname{deg} \Phi_{2}=L^{2} \Phi_{2}=3 L^{3}+c_{1}(\mathcal{V})\left(5 K_{Y}+3 c_{1}(\mathcal{V})\right)>3 d$, provided that $(Y, \mathcal{V})$ is not any of the exceptions listed in Propositions 5.2 and 5.3, while $\operatorname{deg} \Phi_{2}=3 d$ in case (2) of Proposition 5.3. Cases (5) of Proposition 5.2 and (3) of Proposition 5.3 lead to (3) and (4) in the statement, respectively, and the precise expression for $\operatorname{deg} \Phi_{2}$ simply follows from the previous discussion of these cases. Exception (2) in Proposition 5.2 gives (1) in the statement; note that this is the pair discussed in Example 4.2 for $m=2$. Exception (1) in Proposition 5.3 gives (2). Finally, exceptions (1), (3), and (4) in Proposition 5.2 do not satisfy the assumption on the generic rank of the second jet map, because $h^{0}(\mathcal{L})=h^{0}(\mathcal{V}) \leq$ 8 in all these cases. 
A similar result can be obtained for fourfold scrolls over surfaces. In this case the expression provided by Theorem 4.1 becomes

$$
\left[\Phi_{2}\right]=\pi^{*}\left(6 K_{Y}+3 c_{1}(\mathcal{V})\right)+3 L,
$$

so we have to look at the effectiveness of $2 K_{Y}+\operatorname{det} \mathcal{V}$, where now $\mathcal{V}$ is of rank 3. Letting $A:=K_{Y}+\operatorname{det} \mathcal{V}$ as before, we get that the analogue of Proposition 5.2 is simply that $A$ is very ample unless $(Y, \mathcal{V})=\left(\mathbb{P}^{2}, \mathcal{O}_{\mathbb{P}^{2}}(1)^{\oplus 3}\right)$. Apart from this case, arguing as in Proposition 5.3, we obtain that $2 K_{Y}+\operatorname{det} \mathcal{V}$ is effective unless $(Y, \operatorname{det} \mathcal{V})$ is as in cases (1), (2) of Proposition 5.3, or $Y$ is a $\mathbb{P}^{1}$-bundle over a smooth curve $B$ and $\mathcal{V}_{f}=\mathcal{O}_{\mathbb{P}^{1}}(1)^{\oplus 3}$ for every fiber $f$ of the bundle projection $Y \rightarrow B$. This immediately leads to a result of the same type as Theorem 5.5.

Consider again a threefold scroll $X=\mathbb{P}(\mathcal{V}) \subset \mathbb{P}^{8}$ satisfying our general assumptions, and let $g$ be its sectional genus. Then (4.3) gives

$$
\operatorname{deg} \Phi_{2}=10(g-1)+c_{1}(\mathcal{V})^{2}-3 c_{2}(\mathcal{V})
$$

Note that, for the scroll $X=\mathbb{P}\left(\mathcal{O}_{\mathbb{P}^{2}}(1) \oplus \mathcal{O}_{\mathbb{P}^{2}}(2)\right)$ discussed in Example 4.2 for $n=3$, the value of $c_{1}(\mathcal{V})^{2}-3 c_{2}(\mathcal{V})$ is 3 . Putting some restriction on the vector bundle $\mathcal{V}$, we get the following characterization.

Proposition 5.6. Let $X \subset \mathbb{P}^{8}$ be a threefold scroll over a smooth surface $Y$, satisfying our general assumptions and such that $c_{1}(\mathcal{V})^{2} \geq 3 c_{2}(\mathcal{V})$. Then either $X=\mathbb{P}\left(\mathcal{O}_{\mathbb{P}^{2}}(1) \oplus \mathcal{O}_{\mathbb{P}^{2}}(2)\right)$ or $\operatorname{deg} \Phi_{2} \geq 10(g-1) \geq 20$.

Proof. Equation (5.2) gives $\operatorname{deg} \Phi_{2} \geq 10(g-1)$, due to the assumption on the Chern classes of $\mathcal{V}$. Now we use classification results for projective manifolds with low sectional genus [6]. Case $g=0$ cannot occur. Actually, by a result of Sato [16, Theorem A], the only possibility would be that $X$ is the Segre product $\mathbb{P}^{2} \times \mathbb{P}^{1} \subset$ $\mathbb{P}^{5}$, which, as noted in Example 2.3, does not satisfy our general assumptions. Suppose $g=1$. Then $(X, \mathcal{L})$ is either a Del Pezzo threefold or a scroll over a smooth curve $W$ of genus 1 . The former case leads to the exception in the statement. Recall that this $X$ is isomorphic to $\mathbb{P}^{3}$ blown up at one point and $\mathcal{L}=\sigma^{*} \mathcal{O}_{\mathbb{P}^{2}}(2) \otimes \mathcal{O}_{X}(-E)$, where $\sigma: X \rightarrow \mathbb{P}^{3}$ is the blowing-up and $E$ is the exceptional divisor. The latter case cannot occur: actually, the fibers of the scroll over $W$ map surjectively to the smooth surface $Y$ via the scroll projection $\pi$, so that $Y=\mathbb{P}^{2}\left[1\right.$, Example 1.4, p. 136]; hence $h^{1}\left(\mathcal{O}_{X}\right)=0$, while, due to the scroll structure over $W$, it should be $h^{1}\left(\mathcal{O}_{X}\right)=1$. Let $g=2$. As in the previous case, we exclude that $(X, \mathcal{L})$ is a scroll over a smooth curve of genus 2 . Then [6, Corollary 3.3] $(X, \mathcal{L})$ is a quadric fibration over $\mathbb{P}^{1}$, and, if we use [7, Theorem, p. 4], the scroll structure over $Y$ allows us to recognize $X$ as the general projection in $\mathbb{P}^{8}$ of the Segre product $\mathbb{F}_{1} \times \mathbb{P}^{1} \subset \mathbb{P}^{9}$, where $\mathbb{F}_{1} \subset \mathbb{P}^{4}$ is the rational cubic scroll [6, Theorem 3.4, case vi]. However, as observed in Example 2.3, this $X$ does not satisfy the assumption $s_{2}=9$. Therefore $g \geq 3$, and the assertion is proved. 
Let $Y \subset \mathbb{P}^{5}$ be any generically 2-regular surface. Using (5.1) and Example 2.2, we can provide an application to the general projection $X \subset \mathbb{P}^{8}$ of the product scroll $X^{o}:=Y \times \mathbb{P}^{1} \subset \mathbb{P}^{11}$. According to [18, Proposition 0.3], the class of $\Phi_{2}(Y)$ is $4 K_{Y}+6 H, H$ being the hyperplane class. Hence $\Phi_{2}\left(X^{o}\right)=\pi^{-1}\left(\Phi_{2}(Y)\right)$ according to Example 2.2. This means that $\Phi_{2}\left(X^{o}\right)$ is a divisor. Projecting generically $X^{o}$ to $\mathbb{P}^{8}$, we expect that the image $X \cong X^{o}$ has the whole $\mathbb{P}^{8}$ as osculating space at the general point. Thus $\Phi_{2}:=\Phi_{2}(X)$ is a divisor containing the projection $\Phi_{2}^{o}$ of $\Phi_{2}\left(X^{o}\right)$ as a component, plus some other components, i.e., $\Phi_{2}=\Phi_{2}^{o}+R$, where $R$ is an effective divisor. In fact, our formula (5.1) says that $\left[\Phi_{2}\right]=3 L+$ $\pi^{*}\left(5 K_{Y}+6 H\right)$, since $\mathcal{V}=H^{\oplus 2}$. As $\Phi_{2}^{o}=\pi^{*}\left(4 K_{Y}+6 H\right)$, this means that $R=3 L+\pi^{*} K_{Y}$. Note that the effectiveness of $R$ is independent of $(Y, H)$. Indeed, $L=Y_{0}+\pi^{*} H$, where $Y_{0}$ is the tautological section of the trivial bundle $\mathcal{O}_{Y}^{\oplus 2}$. Hence $R=3 Y_{0}+\pi^{*}\left(K_{Y}+3 H\right)$, and $K_{Y}+3 H$ is effective, $Y$ being generically 2 -regular. Since $X$ was obtained by projecting $X^{o}$ from a general $\mathbb{P}^{2} \subset \mathbb{P}^{11}$, the above conclusion can be converted to provide information on $X^{o}$. In particular, it says that the part of $\Phi_{2}$ coming from the osculating spaces to $X^{o} \subset \mathbb{P}^{11}$ which meet a general plane is a surface of degree $\left(3 L+\pi^{*} K_{Y}\right) L^{2}=3 d+2 H K_{Y}$.

\section{The Case $k=2$ : Formulas for Threefold Scrolls OVer SURFACES}

Let $X \subset \mathbb{P}^{N}$ be an $n$-dimensional scroll over a smooth surface $Y$, satisfying our general assumptions. Then (2.10) gives (4.1), which we rewrite for the convenience of the reader:

$$
c\left(\mathcal{E}_{2}\right)=\pi^{*} c\left(\mathcal{V}^{\vee}\right) \pi^{*} c\left(\mathcal{V}^{\vee} \otimes T_{Y}\right) c\left(\pi^{*} S^{2} T_{Y} \otimes \mathcal{L}^{-1}\right) .
$$

Recall that, according to Theorem 2.5, to determine the class of $\Phi_{2}$ we have to take the inverse of the expression in (4.1). Recall that here both $\mathcal{V}^{\vee}$ and $\mathcal{V}^{\vee} \otimes T_{Y}$ are vector bundles over a surface. Hence,

$$
c\left(\mathcal{V}^{\vee}\right)^{-1}=1+v_{1}+\left[v_{1}^{2}-v_{2}\right]
$$

where, for shortness, we have set $v_{i}=c_{i}(\mathcal{V})$. On the other hand, let $c_{i}=c_{i}\left(T_{Y}\right)$. Then computing the Chern classes of $\mathcal{V}^{\vee} \otimes T_{Y}$, we get

$$
\begin{aligned}
& c_{1}\left(\mathcal{V}^{\vee} \otimes T_{Y}\right)=-2 v_{1}+(n-1) c_{1} \\
& c_{2}\left(\mathcal{V}^{\vee} \otimes T_{Y}\right)=v_{1}^{2}+2 v_{2}-(2 n-3) v_{1} c_{1}+\left(\begin{array}{c}
n-1 \\
2
\end{array}\right) c_{1}^{2}+(n-1) c_{2} .
\end{aligned}
$$

Thus,

$$
\begin{aligned}
c\left(\mathcal{V}^{\vee} \otimes T_{Y}\right)^{-1}= & 1+\left[2 v_{1}-(n-1) c_{1}\right] \\
& +\left[3 v_{1}^{2}-2 v_{2}-(2 n-1) v_{1} c_{1}+\left(\begin{array}{l}
n \\
2
\end{array}\right) c_{1}^{2}-(n-1) c_{2}\right] .
\end{aligned}
$$


As to the last factor in (4.1), note that $\pi^{*} S^{2} T_{Y} \otimes \mathcal{L}^{-1}$ is a vector bundle of rank 3 on $X$. First of all, the vector bundle $S^{2} T_{Y}$ has total Chern class

$$
c\left(S^{2} T_{Y}\right)=1+3 c_{1}+\left(2 c_{1}^{2}+4 c_{2}\right) .
$$

So, letting $L=c_{1}(\mathcal{L})$ and $C_{i}=\pi^{*} c_{i}=\pi^{*} c_{i}\left(T_{Y}\right)$, we obtain

$$
\begin{aligned}
c\left(\pi^{*} S^{2} T_{Y} \otimes \mathcal{L}^{-1}\right)= & 1+\left[3 C_{1}-3 L\right]+\left[2 C_{1}^{2}+4 C_{2}-6 C_{1} L+3 L^{2}\right] \\
& -\left[\left(2 C_{1}^{2}+4 C_{2}\right) L-3 C_{1} L^{2}+L^{3}\right] .
\end{aligned}
$$

Computing the inverse of this total Chern class is very difficult in general. Let us do it for threefold scrolls. So from now on in this section we let $n=3$. Recall that for a vector bundle $B$ of rank 3 with $b_{i}=c_{i}(B)$ we have

$$
c(B)^{-1}=1-b_{1}+\left[b_{1}^{2}-b_{2}\right]+\left[-b_{1}^{3}+2 b_{1} b_{2}-b_{3}\right] .
$$

Thus, if $n=3$, the inverse of (6.3) is

$$
\begin{aligned}
c\left(\pi^{*} S^{2} T_{Y} \otimes \mathcal{L}^{-1}\right)^{-1}= & 1+3\left[L-C_{1}\right]+\left[6 L^{2}+7 C_{1}^{2}-12 C_{1} L-4 C_{2}\right] \\
& +5\left[2 L^{3}+7 C_{1}^{2} L-4 C_{2} L-6 C_{1} L^{2}\right] .
\end{aligned}
$$

If we let $V_{i}=\pi^{*} v_{i}=\pi^{*} c_{i}(\mathcal{V})$, the product of the three factors in (4.1) gives the following expression:

$$
\begin{aligned}
c\left(\mathcal{E}_{2}\right)^{-1}= & 1+\left[3 L+3 V_{1}-5 C_{1}\right] \\
+ & {\left[6 L^{2}+9 V_{1} L-18 C_{1} L+6 V_{1}^{2}\right.} \\
& \left.-3 V_{2}-16 C_{1} V_{1}+16 C_{1}^{2}-6 C_{2}\right] \\
& +\left[10 L^{3}-42 C_{1} L^{2}+18 V_{1} L^{2}+68 C_{1}^{2} L\right. \\
& \left.\quad-26 C_{2} L-57 C_{1} V_{1} L+18 V_{1}^{2} L-9 V_{2} L\right] .
\end{aligned}
$$

In particular, if $\left[\Phi_{2}\right]$ has codimension $\ell=n=3$, then $X$ has finitely many flexes, their number being given by

$$
\operatorname{deg} \Phi_{2}=19 d+68 c_{1}^{2}-26 c_{2}-99 c_{1} v_{1}+27 v_{1}^{2},
$$

which fits with the computations already done when $Y$ is an abelian surface (3.2).

We shall now apply the formulas to threefold scrolls $X$ over some special surfaces $Y$. According to (2.12), we should consider $X \subset \mathbb{P}^{N}$ with $N=10,9,8$, but in view of the discussion in Section 5, we need only consider the cases $N=10$ and 9. For some of the needed computations it is better to recall that $X=\mathbb{P}(\mathcal{V})$, where $\mathcal{V}=\pi_{*} \mathcal{L}$ is a very ample vector bundle of rank 2 on $Y$. Then it follows 
that $K_{X}=-2 \mathcal{L}+\pi^{*}\left(K_{Y}+\operatorname{det} \mathcal{V}\right)$, where $\pi: X \rightarrow Y$ is the scroll projection. Moreover, $L^{2}=L V_{1}-V_{2}$, where $V_{i}=\pi^{*} c_{i}(\mathcal{V})$, by the Chern-Wu relation. In particular, this gives $L^{2} \pi^{*} D=L V_{1} \pi^{*} D=c_{1}(\mathcal{V}) D$ for any divisor $D$ on $Y$.

First of all, consider $X:=\mathbb{P}\left(\mathcal{O}_{\mathbb{P}^{2}}(2)^{\oplus 2}\right) \subset \mathbb{P}^{11}$. Note that $X$ is the Segre product of the Veronese surface in $\mathbb{P}^{5}$ with $\mathbb{P}^{1}$. Hence $\Phi_{2}(X)=\varnothing$, by what we said at the end of Example 2.2 combined with [18, Proposition 3.8]. This could lead to doubts concerning formula (6.5). However, note that here $N=11$ instead of 10 . So the formula does not apply to $X$, but it applies to its general projection in $\mathbb{P}^{10}$, for which we get $\operatorname{deg} \Phi_{2}=6$. Rephrasing this in terms of the linearly normal scroll $X \subset \mathbb{P}^{11}$ gives the following result:

Corollary 6.1. Through a general point of $\mathbb{P}^{11}$ there pass 6 second-order osculating spaces of the Segre product $S \times \mathbb{P}^{1}$, where $S$ is the Veronese surface in $\mathbb{P}^{5}$.

A nice result concerning scrolls $X \subset \mathbb{P}^{10}$, derived from formula (6.5), is the following:

Proposition 6.2. Let $X \subset \mathbb{P}^{10}$ be a threefold scroll over $\mathbb{P}^{2}$, satisfying our general assumptions. Then $\Phi_{2}(X) \neq \varnothing$.

Proof. If $X$ is uninflected, then Theorem 2.5 applies, and hence the expression in formula (6.5) is equal to 0 . As $Y=\mathbb{P}^{2}$, if we let $(x, y)=\left(c_{1}(\mathcal{V}), c_{2}(\mathcal{V})\right)$, then (6.5) becomes $\operatorname{deg} \Phi_{2}=46 x^{2}-297 x+534-19 y$. Then $(x, y)$ must be an integral point of the parabola $C \subset \mathbb{A}^{2}$ defined by $y=\frac{1}{19}\left(46 x^{2}-297 x+534\right)$. On the other hand, the point $(x, y)$ must lie in the region $R \subset \mathbb{A}^{2}$ defined by $y \leq x^{2}-8$, since $x^{2}-y=d \geq \operatorname{codim}_{\mathbb{1 0} 10}(X)+1=8$. But an immediate check shows that there are no integral points on the $\operatorname{arc} R \cap C$.

In the following we assume that our threefold scrolls $X \subset \mathbb{P}^{N}$ satisfy the general assumptions of the discussion leading to the formulas in Section 4.

Now consider scrolls $X \subset \mathbb{P}^{9}$. Here $\ell=2$, so that $\Phi_{2}$ is a 1 -cycle, whose class is the second term in (6.4). Dotting it with $L$ and taking into account the Chern-Wu relation, we get the following expression for its degree:

$$
\operatorname{deg} \Phi_{2}=9 d+12 c_{1}(\mathcal{V})^{2}+34 K_{Y} c_{1}(\mathcal{V})+16 K_{Y}^{2}-6 c_{2}\left(T_{Y}\right) .
$$

First suppose $Y=\mathbb{P}^{2}$. In this case, if we let $v=c_{1}(\mathcal{V})$, (6.6) becomes

$$
\operatorname{deg} \Phi_{2}=9 d+6 v(2 v-17)+126 .
$$

This allows us to prove an analogue of Proposition 6.2.

Proposition 6.3. Let $X \subset \mathbb{P}^{9}$ be a threefold scroll over $\mathbb{P}^{2}$, satisfying our general assumptions. Then $\Phi_{2}(X) \neq \varnothing$.

Proof. If $\Phi_{2}(X)=\varnothing$, then $\operatorname{deg} \Phi_{2}=0$, so that (6.7), after dividing by 3 , gives

$$
3(d+14)=2 v(17-2 v) .
$$


Note that $X$ has degree $d \geq \operatorname{codim}_{\mathbb{p} 9}(X)+1=7$. Hence the left-hand-side term is greater than or equal to 63 . Then looking at the right-hand-side term, we conclude that $v$ can only be $3,4,5$, or 6 . However, $v=5$ is not possible; otherwise the right-hand-side term would not be divisible by 3 . If $v=3$, then the equation above gives $d=8$; hence $8=v^{2}-c_{2}(\mathcal{V})=9-c_{2}(\mathcal{V})$, i.e., $c_{2}(\mathcal{V})=1$, but this would imply that $\mathcal{V}=\mathcal{O}_{\mathbb{P}^{2}}(1)^{\oplus 2}$ [2, Theorem 11.1.3], which is clearly not compatible with $v=3$. If $v=6$, then the equation above gives $d=6$. On the other hand, recalling what we said in the proof of Proposition 3.1, the curve genus of $(X, \mathcal{L})$ is $g=g\left(\mathbb{P}^{2}, \mathcal{O}_{\mathbb{P}^{2}}(v)\right)=10$, since $v=6$. But this implies that the general curve section of $X$ is a plane curve, which is clearly impossible. The only remaining possibility is $v=4$, which gives $g=3, d=10$, and $c_{2}(\mathcal{V})=6$. These invariants characterize the Bordiga scrolls of degree 10, but, as observed in Example 2.6, they do not satisfy our general assumptions.

The next result takes care of the case $Y=\mathbb{F}_{e}$, a Segre-Hirzebruch surface.

Proposition 6.4. Let $X \subset \mathbb{P}^{9}$ be a threefold scroll over $\mathbb{F}_{e}(e \geq 0)$. Then $\Phi_{2}(X) \neq \varnothing$, except possibly if $\mathcal{V}$ is uniform of type $(1,1)$ on the fibers $\mathbb{F}_{e}$, and

$$
9 d-32=20(b-e),
$$

where $b$ is the degree of $\mathcal{V}$ restricted to a minimal section of $\mathbb{F}_{e}$.

Proof. Let $s$ and $f$ be a section of minimal self-intersection $\left(s^{2}=-e\right)$ and a fiber of $\mathbb{F}_{e}$, respectively. Since the classes of $s$ and $f$ generate $\operatorname{Pic}\left(\mathbb{F}_{e}\right)$, we can write $\operatorname{det} \mathcal{V}=a s+b f$, where $a=\operatorname{deg} \mathcal{V}_{f} \geq \mathrm{rk} \mathcal{V}=2$, equality implying that $\mathcal{V}_{f}=\mathcal{O}_{\mathbb{P}^{1}}(1)^{\oplus 2}$ for every fiber $f$. Moreover, $b-a e=(a s+b f) s=\operatorname{deg} \mathcal{V}_{s} \geq 2$. Recalling that $K_{\mathbb{F}_{e}}=-2 s-(2+e) f,(6.6)$ gives $\operatorname{deg} \Phi_{2}=9 d+12(2 b-a e) a+$ $34(a e-2 a-2 b)+104$. So, if $\Phi_{2}(X)=\varnothing$, then $(a, b)$ must be an integral point in the region $R$ of the affine plane defined by $a \geq 2, b \geq e a+2$, lying on the hyperbola $\gamma$ with equation

$$
12 e a^{2}-24 a b+34(2-e) a+68 b-(9 d+104)=0 .
$$

Let us draw a picture. The asymptotes of $\gamma$ are the lines parallel to the $a$-axis and to the line of equation $b=(e / 2) a$ passing through the center $A$, which has coordinates $\left(\frac{17}{6}, \frac{17}{6}+\frac{17}{12} e\right)$. Note that the second asymptote cuts the line $a=2$ at the point $T=\left(2, e+\frac{17}{6}\right)$. On the other hand, the hyperbola $\gamma$ cuts the line $a=2$ at the point $B=(2, e+(9 d-32) / 20)$. Note that, as a consequence of (6.8), $d$ must be even. On the other hand, $d \geq \operatorname{codim}_{\mathbb{P} 9}(X)+1=7$, as already observed. Hence either $d=8$ or $d \geq 10$. Suppose that $d=8$. Then $(X, \mathcal{L})$ has $\Delta$-genus $\leq 1$ (since our $X \subset \mathbb{P}^{9}$ is not necessarily linearly normal). This implies that it is either a scroll over $\mathbb{P}^{1}$ or a Del Pezzo threefold [6, Proposition 2.4]. The former case cannot occur because our $X$ has Picard number 3. The latter case also gives a contradiction, since the only Del Pezzo threefold of degree 8 is the pair 
$\left(\mathbb{P}^{3}, \mathcal{O}_{\mathbb{P}^{3}}(2)\right)$. Therefore $d \geq 10$. This fact implies that the second coordinate of $B$ is bigger than that of $T$. Now, looking at the position of the region $R$, we can see that $\gamma \cap R$ does not contain integral points on the branch lying in the half plane $a>2$. As a consequence, the integral points on $\gamma \cap R$ we are looking for are confined between the line $a=2$ and the vertical asymptote $a=\frac{17}{6}$. Since this value is smaller than 3 , it turns out that they consist of the single point $B$. Therefore $a=2$, which implies that $\mathcal{V}$ is uniform of type $(1,1)$ on the fibers $f$, and $b=e+(9 d-32) / 20$, which leads to the numerical condition in the statement.

By the same method we can produce a result taking care of another interesting class of surfaces, namely, products of curves. Since the case $Y=\mathbb{F}_{0}=\mathbb{P}^{1} \times \mathbb{P}^{1}$ has already been considered, we will assume that not both factors are rational curves.

Proposition 6.5. Let $X \subset \mathbb{P}^{9}$ be a threefold scroll over a surface $Y$ which is the product of two smooth curves, not both rational. Then $\Phi_{2}(X) \neq \varnothing$, except possibly for the following case: $Y=B \times \mathbb{P}^{1}$, where $B$ has genus $q \geq 1, V$ is uniform of type $(1,1)$ on the fibers of the first projection, and

$$
9 d+32(q-1)=20 b,
$$

where $b$ is the degree of $\mathcal{V}$ restricted to the fibers of the second projection (in particular, $b \geq 5$ ).

Proof. Set $Y=C_{1} \times C_{2}$, and let $g_{i}$ be the genus of the curve $C_{i}, i=1,2$. Up to renaming we can suppose that $g_{1} \geq g_{2}$. Since $K_{Y}^{2}=8\left(g_{1}-1\right)\left(g_{2}-1\right)$, $c_{2}\left(T_{Y}\right)=4\left(1-g_{1}\right)\left(1-g_{2}\right)$, and $\operatorname{deg} \mathcal{V}_{C_{i}}>0$, (6.6) immediately implies that $\operatorname{deg} \Phi_{2} \geq 9 d+12 c_{1}(\mathcal{V})^{2}>0$ if $g_{2} \geq 1$. So, we can put $C_{2}=\mathbb{P}^{1}$. In other words, it only remains to consider the case when $Y=B \times \mathbb{P}^{1}$, where $B$ has genus $q \geq 1$. If we let $f$ and $s$ denote the fibers of the first and the second projection, respectively, $\operatorname{det} \mathcal{V}$ is numerically equivalent to $a s+b f$, where $a=\operatorname{deg} \mathcal{V}_{f} \geq$ $\mathrm{rk} \mathcal{V}=2$, equality implying that $\mathcal{V}_{f}=\mathcal{O}_{\mathbb{P}^{1}}(1)^{\oplus 2}$ for every fiber $f$. Moreover, $b=\operatorname{deg} \mathcal{V}_{s} \geq 5$, since there are no irrational surface scrolls of degree less than 5 . Then (6.6) gives $\operatorname{deg} \Phi_{2}=9 d+24 a b+68(q-1) a-68 b+104(1-q)$. So, if $\Phi_{2}(X)=\varnothing$, then $(a, b)$ must be an integral point in the region $R$ of the affine plane defined by $a \geq 2, b \geq 5$, lying on the hyperbola $\gamma$ with equation

$$
24 a b+68(q-1) a-68 b+9 d-104(q-1)=0 .
$$

The asymptotes of $\gamma$ are the parallel lines to the $a$-axis and to the $b$-axis passing through the center $A$, which has coordinates $\left(\frac{17}{6}, \frac{17}{6}(1-q)\right)$. On the other hand, $\gamma$ cuts the line $a=2$ at the point $B$ whose second coordinate is $\frac{1}{20}(9 d+32(q-1))$, which is bigger than that of $A$. Then the integral points on $\gamma \cap R$ we are looking for are confined between the line $a=2$ and the vertical asymptote, and since the abscissa of $A$ is smaller than 3 , they consist of the single point $B$. Therefore 
$a=2$, which implies that $\mathcal{V}$ is uniform of type $(1,1)$ on the fibers $f$, and $b=$ $\frac{1}{20}(9 d+32(q-1))$, which leads to the numerical condition in the statement.

If $Y$ is an abelian surface, then (6.6) gives $\operatorname{deg} \Phi_{2}=9 d+12(2 g-2)$, in accordance with the general formula in Proposition 3.1. For $Y$ a K3 surface, (6.6) gives $\operatorname{deg} \Phi_{2}=3(3 d+4(2 g-2)-48)$. Recall that $d \geq 7$ as already observed. Let $S$ be a general surface section of $X$, and look at the reduction $(Y$, det $\mathcal{V})$ of $\left(S, \mathcal{L}_{S}\right)$. The general curve $C \in|\operatorname{det} \mathcal{V}|$ is a canonical curve of degree $d+c_{2}(\mathcal{V}) \geq 8$; hence $g(C) \geq 5$. Therefore $g \geq 5$ and then $\operatorname{deg} \Phi_{2} \geq 15$. Though this is a rough inequality, it is enough to conclude the following result.

Proposition 6.6. Let $X \subset \mathbb{P}^{9}$ be a threefold scroll over a $\mathrm{K} 3$ surface. Then $\Phi_{2}(X) \neq \varnothing$.

We can prove a similar result for $Y$ a minimal surface of Kodaira dimension 1, in two cases: when the base curve of the elliptic fibration has positive genus, and when the base is $\mathbb{P}^{1}$ provided that $Y$ is an elliptic quasi-bundle in the sense of Serrano [17, Definition 1.1 and Lemma 1.5].

\section{The CASE $k=2$ : Formulas For Fourfold SCrolls OVER THREEFOLDS}

Let $X \subset \mathbb{P}^{N}$ be a fourfold scroll over a smooth threefold $Y$, satisfying our general assumptions. Again, according to Theorem 2.5, to determine the class of $\Phi_{2}$ we have to take the inverse of the expression in (4.1). Now both $\mathcal{V}^{\vee}$ and $\mathcal{V}^{\vee} \otimes T_{Y}$ are vector bundles over a threefold. Hence (6.1) has to be replaced with

$$
c\left(\mathcal{V}^{\vee}\right)^{-1}=1+v_{1}+\left[v_{1}^{2}-v_{2}\right]+\left[v_{1}^{3}-2 v_{1} v_{2}\right],
$$

where $v_{i}=c_{i}(\mathcal{V})$. In the following, as in Section 6 we set also $c_{i}=c_{i}\left(T_{Y}\right)$. On the other hand, by using the splitting principle confined to $\mathcal{V}^{\vee}$, we easily get the following expressions for the Chern classes of $\mathcal{V}^{\vee} \otimes T_{Y}$. They are

$$
\begin{aligned}
c_{1}\left(\mathcal{V}^{\vee} \otimes T_{Y}\right)= & -3 v_{1}+2 c_{1}, \\
c_{2}\left(\mathcal{V}^{\vee} \otimes T_{Y}\right)= & 3 v_{1}^{2}+3 v_{2}-5 c_{1} v_{1}+c_{1}^{2}+2 c_{2}, \\
c_{3}\left(\mathcal{V}^{\vee} \otimes T_{Y}\right)= & -v_{1}^{3}-6 v_{1} v_{2}+4 c_{1}\left(v_{1}^{2}+v_{2}\right)-\left(2 c_{1}^{2}+4 c_{2}\right) v_{1} \\
& +2 c_{1} c_{2}+2 c_{3} .
\end{aligned}
$$

Thus, the analogue of (6.2) for $n=4$ is now the following:

$$
\begin{gathered}
c\left(\mathcal{V}^{\vee} \otimes T_{Y}\right)^{-1}=1+\left[3 v_{1}-2 c_{1}\right]+\left[6 v_{1}^{2}-3 v_{2}-7 c_{1} v_{1}+3 c_{1}^{2}-2 c_{2}\right] \\
+\left[10 v_{1}^{3}-12 v_{1} v_{2}-16 c_{1} v_{1}^{2}+8 c_{1} v_{2}\right. \\
\left.+12 c_{1}^{2} v_{1}-8 c_{2} v_{1}-4 c_{1}^{3}+6 c_{1} c_{2}-2 c_{3}\right] .
\end{gathered}
$$


As to the last factor in (4.1), note that $\pi^{*} S^{2} T_{Y} \otimes \mathcal{L}^{-1}$ is a vector bundle of rank 6 on $X$. First of all, the vector bundle $S^{2} T_{Y}$ has total Chern class

$$
c\left(S^{2} T_{Y}\right)=1+4 c_{1}+5\left(c_{1}^{2}+c_{2}\right)+2 c_{1}^{3}+11 c_{1} c_{2}+7 c_{3} .
$$

So, letting $L=c_{1}(\mathcal{L})$ and $C_{i}=\pi^{*} c_{i}=\pi^{*} c_{i}\left(T_{Y}\right)$ as in Section 6, we obtain

$$
\begin{aligned}
c\left(\pi^{*} S^{2} T_{Y} \otimes \mathcal{L}^{-1}\right)=1+ & {\left[4 C_{1}-6 L\right]+\left[5\left(C_{1}^{2}+C_{2}\right)-20 C_{1} L+15 L^{2}\right] } \\
+ & {\left[2 C_{1}^{3}+11 C_{1} C_{2}+7 C_{3}\right.} \\
& \left.-20\left(C_{1}^{2}+C_{2}\right) L+40 C_{1} L^{2}-20 L^{3}\right] \\
+ & -3\left(2 C_{1}^{3}+11 C_{1} C_{2}+7 C_{3}\right) L \\
& \left.+30\left(C_{1}^{2}+C_{2}\right) L^{2}-40 C_{1} L^{3}+15 L^{4}\right] .
\end{aligned}
$$

Recall that for a vector bundle $B$ on a fourfold, with $b_{i}=c_{i}(B)$, we have

$$
\begin{aligned}
c(B)^{-1}= & 1-b_{1}+\left[b_{1}^{2}-b_{2}\right]+\left[-b_{1}^{3}+2 b_{1} b_{2}-b_{3}\right] \\
& +\left[b_{1}^{4}-3 b_{1}^{2} b_{2}+2 b_{1} b_{3}+b_{2}^{2}-b_{4}\right] .
\end{aligned}
$$

Thus, since $n=4$, the inverse of $(7.1)$ is

$$
\begin{aligned}
c\left(\pi^{*} S^{2} T_{Y} \otimes \mathcal{L}^{-1}\right)^{-1}=1+ & {\left[6 L-4 C_{1}\right]+\left[21 L^{2}-28 C_{1} L+11 C_{1}^{2}-5 C_{2}\right] } \\
+ & {\left[56 L^{3}-112 C_{1} L^{2}+88 C_{1}^{2} L\right.} \\
& \left.-40 C_{2} L-26 C_{1}^{3}+29 C_{1} C_{2}-7 C_{3}\right] \\
+ & {\left[126 L^{4}-336 C_{1} L^{3}+396 C_{1}^{2} L^{2}\right.} \\
& \left.-180 C_{2} L^{2}-234 C_{1}^{3} L+261 C_{1} C_{2} L-63 C_{3} L\right] .
\end{aligned}
$$

Now, if we let $V_{i}=\pi^{*} v_{i}=\pi^{*} c_{i}(\mathcal{V})$, the product of the inverses of the three factors in (4.1) (which involves a tedious computation) will give the following expression for $\Phi_{2}$, according to whether $\ell=2,3,4$, respectively (for the case $\ell=1$, see Section 4):

$$
\begin{aligned}
{\left[\Phi_{2}\right]=} & 21 L^{2}+24 V_{1} L-40 C_{1} L+10 V_{1}^{2}-4 V_{2}-25 C_{1} V_{1}+22 C_{1}^{2}-7 C_{2}, \\
{\left[\Phi_{2}\right]=} & 56 L^{3}-154 C_{1} L^{2}+84 V_{1} L^{2}+162 C_{1}^{2} L-52 C_{2} L-166 C_{1} V_{1} L \\
& +60 V_{1}^{2} L-24 V_{2} L+20 V_{1}^{3}-20 V_{1} V_{2}-65 C_{1} V_{1}^{2}+26 C_{1} V_{2} \\
& +95 C_{1}^{2} V_{1}-30 C_{2} V_{1}-64 C_{1}^{3}+53 C_{1} C_{2}-9 C_{3}, \\
{\left[\Phi_{2}\right]=} & 126 L^{4}+224 V_{1} L^{3}-448 C_{1} L^{3}-84 V_{2} L^{2}+210 V_{1}^{2} L^{2}-637 C_{1} V_{1} L^{2} \\
& +683 C_{1}^{2} L^{2}-222 C_{2} L^{2}+694 C_{1}^{2} V_{1} L-220 C_{2} V_{1} L \\
& +120 V_{1}^{3} L-120 V_{1} V_{2} L-430 C_{1} V_{1}^{2} L+172 C_{1} V_{2} L \\
& -518 C_{1}^{3} L+433 C_{1} C_{2} L-75 C_{3} L .
\end{aligned}
$$


To compute the degree of $\Phi_{2}$ in all cases we have to dot the above expressions with $L^{4-\ell}$. Notice that by using the Chern-Wu relation we can avoid the appearance of the term $v_{1} v_{2}$ in all expressions. Recall that $c_{i}=c_{i}\left(T_{Y}\right)$ and $v_{i}=c_{i}(\mathcal{V})$. First of all, if $\Phi_{2}$ has codimension $\ell=1$, then $\operatorname{deg} \Phi_{2}$ is given by (4.2), which, in the present case $(n, m)=(4,3)$, becomes

$$
\operatorname{deg} \Phi_{2}=8 d+2 v_{1}^{3}-6 c_{1} v_{1}^{2}+6 c_{1} v_{2}
$$

If $\ell=2$, then $\Phi_{2}$ is a 2 -cycle of degree

$$
\operatorname{deg} \Phi_{2}=35 d+20 v_{1}^{3}-65 c_{1} v_{1}^{2}+40 c_{1} v_{2}+22 c_{1}^{2} v_{1}-7 c_{2} v_{1}
$$

If $\ell=3$, then $\Phi_{2}$ is a 1 -cycle of degree

$$
\begin{aligned}
\operatorname{deg} \Phi_{2}= & 120 d+100 v_{1}^{3}-385 c_{1} v_{1}^{2}+180 c_{1} v_{2}+257 c_{1}^{2} v_{1} \\
& -82 c_{2} v_{1}-64 c_{1}^{3}+53 c_{1} c_{2}-9 c_{3} .
\end{aligned}
$$

Finally, if the codimension $\ell$ of $\Phi_{2}$ is 4 , then $X$ has finitely many flexes, and their number is given by

$$
\begin{aligned}
\operatorname{deg} \Phi_{2}= & 340 d+340 v_{1}^{3}-1515 c_{1} v_{1}^{2}+620 c_{1} v_{2}+1377 c_{1}^{2} v_{1} \\
& -442 c_{2} v_{1}-518 c_{1}^{3}+433 c_{1} c_{2}-75 c_{3} .
\end{aligned}
$$

Note that, when $Y$ is an abelian threefold, the previous expressions of $\left[\Phi_{2}\right]$ are consistent with the result provided by Proposition 3.3.

Here is an application of our formulas.

Theorem 7.1. Let $X \subset \mathbb{P}^{N}$ be a fourfold scroll over $\mathbb{P}^{3}$, satisfying our general assumptions. Then $\Phi_{2}(X) \neq \varnothing$.

Proof. Clearly, $\Phi_{2}(X)$ cannot be a divisor, by Remark 4.3. So $\ell \geq 2$. Suppose that $\Phi_{2}(X)=\varnothing$; then $\operatorname{deg} \Phi_{2}=0$. For $Y=\mathbb{P}^{3}$ we have $c_{1}=c_{3}=4, c_{2}=6$. Set $x=v_{1}, y=v_{2}$. Then, according to the previous formulas, $\operatorname{deg} \Phi_{2}$ is given by

$$
\begin{aligned}
& 5\left(7 d+4 x^{3}-52 x^{2}+32 y+62 x\right), \\
& 20\left(6 d+5 x^{3}-77 x^{2}+36 y+181 x-143\right), \\
& 20\left(17 d+17 x^{3}-303 x^{2}+124 y+969 x-1153\right),
\end{aligned}
$$

according to whether $\ell=2,3,4$, respectively. First, consider the case $\ell=4$. Condition $\operatorname{deg} \Phi_{2}=0$ gives

$$
17 d+124 y=-17 x^{3}+303 x^{2}-969 x+1153 .
$$


Note that the left-hand side is positive (since $d>0$ and $\mathcal{V}$ is ample), while the right-hand side is negative for $x \gg 0$. In fact, for it to be positive, we need $x \leq 14$. On the other hand, $x \geq 2$ by Remark 5.1 (A1). Now, by using the Chern-Wu relation, which in the present case says that $d=x^{3}-2 x y$, equation (7.2) can be rewritten as

$$
34 x^{3}-34 x y-303 x^{2}+124 y+969 x-1153=0,
$$

which gives

$$
y=\frac{34 x^{3}-303 x^{2}+969 x-1153}{2(17 x-62)} .
$$

However, a close check shows that this expression does not produce a positive integer for any integer $x$ such that $2 \leq x \leq 14$. Therefore $\ell \neq 4$. Case $\ell=3$ can be ruled out with a similar procedure. In this case, the analogue of $(7.2)$ is

$$
6 d+36 y=-5 x^{3}+77 x^{2}-181 x+143,
$$

and the positivity of the left-hand side implies that $x \leq 12$. Noting that $x$ cannot be 3 , we have that the analogue of (7.3) is

$$
y=\frac{11 x^{3}-77 x^{2}+181 x-143}{12(x-3)},
$$

and a direct check shows that $y$ is not an integer for $x=2$ and for any integer $x$ with $4 \leq x \leq 12$. So the only case left is $\ell=2$. Here the analogue of (7.2) is

$$
7 d+32 y=-2 x\left(2 x^{2}-26 x+31\right),
$$

and the positivity of the left-hand side implies that $x \leq 11$. On the other hand, the analogue of $(7.3)$ is

$$
y=\frac{x\left(11 x^{2}-52 x+62\right)}{2(7 x-16)} .
$$

For $x$ in the range $2 \leq x \leq 11$, we can see that this expression provides a positive integer only for $x=4$ and 8 . However, for $x=8$, condition $y<x^{2} / 2$, coming from the Chern-Wu relation, is not satisfied. The case $x=4$ gives $y=5$. Clearly, if $\mathcal{V}$ is a rank 2 vector bundle with Chern classes $v_{1}=4, v_{2}=5$, then it is indecomposable. Moreover, since $v_{1}=4$, the canonical bundle formula shows that $K_{X}=-2 \mathcal{L}$. Thus $X$ is a Fano fourfold of index 2. In particular, $X$ is a Fano bundle in the terminology of [21], and since $\mathcal{V}$ is indecomposable, it follows from [21, Theorem 2.1] that $\mathcal{V}$ is a twist of the null correlation bundle $N$ fitting into an exact sequence

$$
0 \rightarrow N \rightarrow T_{\mathbb{P} 3}(-1) \rightarrow \mathcal{O}_{\mathbb{P}^{3}}(1) \rightarrow 0
$$


[14, p. 77]. In fact, from $\left(v_{1}, v_{2}\right)=(4,5)$, we deduce that $\mathcal{V}=N(2)$. Dualizing the above sequence and using the fact that $N \cong N^{\vee}$, we see that $N(1)$ is a quotient of $\wedge^{2}\left(T_{\mathbb{P}^{3}}(-1)\right)$, hence spanned. Therefore, $N(2)=N(1) \otimes \mathcal{O}_{\mathbb{P}^{3}}(1)$ is very ample. However, $h^{0}(N(2))=16$; hence for $X=\mathbb{P}(N(2))$ the tautological line bundle $\mathcal{L}$ provides an embedding into $\mathbb{P}^{15}$, so that, if our general assumptions are satisfied, we should have $\ell=3$ instead of 2 . To have $\ell=2$, our scroll $X$ would be a general projection in $\mathbb{P}^{14}$ of this scroll, say, $X^{o}$. However, in this case $\Phi_{2}(X)$ could not be empty. Actually, for some $x \in X^{o}$, the osculating space to $X^{o}$ at $x$ contains the center of projection; hence the image of $x$ in $X$ belongs to $\Phi_{2}(X)$.

Now let $Y=\mathbb{Q}^{3} \subset \mathbb{P}^{4}$ be a smooth quadric hypersurface, and let $h=$ $c_{1}\left(\mathcal{O}_{\mathbb{Q}^{3}}(1)\right)$. Then $\left(c_{1}, c_{2}, c_{3}\right)=\left(3 h, 4 h^{2}, 2 h^{3}\right)$ and $h^{3}=2$. Letting $v_{1}=x h$ and $v_{2}=y\left(h^{2} / 2\right)$ (recall that $h^{2} / 2$ generates 1 -cycles) for some positive integers $x, y$ and arguing in a similar way, we get the following result.

Theorem 7.2. Let $X \subset \mathbb{P}^{N}$ be a fourfold scroll over $\mathbb{Q}^{3}$, satisfying our general assumptions. Then $\Phi_{2}(X) \neq \varnothing$.

Acknowledgements. The first author would like to thank the MiUR of the Italian Government for support received in the framework of the PRIN "Algebraic varieties etc." (Cofin 2006 and 2008) as well as the University of Milano (FIRST 2006 and 2007) for making this collaboration possible. The second author wants to thank the NILS Project and the Spanish Ministry of Education (projects MTM 2007-61124 and MTM 2009-06964) for the funds received supporting this research.

\section{REFERENCES}

[1] W. Barth, C. Peters, and A. Van de Ven, Compact Complex Surfaces, Ergebnisse der Mathematik und ihrer Grenzgebiete (3) [Results in Mathematics and Related Areas (3)], vol. 4, Springer-Verlag, Berlin, 1984. MR749574 (86c:32026)

[2] M.C. BeltrametTi and A.J. Sommese, The Adjunction Theory of Complex Projective Varieties, de Gruyter Expositions in Mathematics, vol. 16, Walter de Gruyter \& Co., Berlin, 1995. MR1318687 (96f:14004)

[3] C. CiliberTO and K. HUleK, A series of smooth irregular varieties in projective space, Ann. Scuola Norm. Sup. Pisa Cl. Sci. (4) 28 (1999), no. 2, 357-380. MR1736233 (2000i:14080)

[4] $\quad$ A bound on the irregularity of abelian scrolls in projective space, Complex Geometry (Göttingen, 2000), Springer, Berlin, 2002, pp. 85-92. MR1922098 (2003f:14049)

[5] W. FULTON, Intersection Theory, 2nd ed., Ergebnisse der Mathematik und ihrer Grenzgebiete. 3. Folge, vol. 2, Springer-Verlag, Berlin, 1998. [Results in Mathematics and Related Areas. 3rd Series. A Series of Modern Surveys in Mathematics]. MR1644323 (99d:14003)

[6] P. IONESCU, Embedded projective varieties of small invariants, Algebraic Geometry, Bucharest 1982 (Bucharest, 1982), Lecture Notes in Math., vol. 1056, Springer, Berlin, 1984, pp. 142186. http://dx.doi.org/10.1007/BFb0071773. MR749942 (85m:14024)

[7] A. LANTERI, Scrolls over surfaces allowing quadric bundle structures over curves are the expected ones, Istit. Lombardo Accad. Sci. Lett. Rend. A 132 (1998), no. 1-2, 3-13 (2000) (English, with Italian summary). MR1869327 (2002j:14008)

[8] A. Lanteri and R. Mallavibarrena, Osculating properties of decomposable scrolls, Math. Nachr. 282 (2009), no. 11, 1548-1566. http://dx.doi.org/10.1002/mana.200610834. MR2573465 (2010k:14099) 
[9] A. Lanteri, R. Mallavibarrena, and R. Piene, Inflectional loci of scrolls, Math. Z. 258 (2008), no. 3, 557-564. http://dx.doi.org/10.1007/s00209-007-0185-5. MR2369044 (2008m:14106)

[10] A. LANTERI and V. NANNOLA, Remarks on the Bordiga scrolls of degree ten, JP J. Algebra Number Theory Appl. 13 (2009), no. 2, 209-219. MR2538053 (2010i:14068)

[11] H. MAEDA, The threefold containing the Bordiga surface of degree ten as a hyperplane section, Math. Proc. Cambridge Philos. Soc. 145 (2008), no. 3, 619-622. http://dx.doi.org/10.1017/S0305004108001461. MR2464780 (2010d:14059)

[12] M.B. Monagan, K.O. Geddes, K.M. Heal, G. Labahn, S.M. Vorkoetter, J. MCCarron, and P. DeMarco, Maple 10 Programming Guide, Maplesoft, Waterloo ON, Canada, 2005.

[13] M. OHNo, Classification of generalized polarized manifolds by their nef values, Adv. Geom. 6 (2006), no. 4, 543-599. http://dx.doi.org/10.1515/ADVGEOM.2006.033. MR2267037 (2008b:14074a)

[14] C. OKoneK, M. Schneider, and H. SPIndler, Vector Bundles on Complex Projective Spaces, Progress in Mathematics, vol. 3, Birkhäuser Boston, Mass., 1980. MR561910 (81b:14001)

[15] R. PIENE and G. SACCHIERO, Duality for rational normal scrolls, Comm. Algebra 12 (1984), no. 9-10, 1041-1066. http://dx.doi.org/10.1080/00927878408823038. MR738534 (86c:14042)

[16] E. SATO, Varieties which have two projective space bundle structures, J. Math. Kyoto Univ. 25 (1985), no. 3, 445-457. MR807491 (87a:14016)

[17] F. SERrano, The Picard group of a quasi-bundle, Manuscripta Math. 73 (1991), no. 1, 63-82. http://dx.doi.org/10.1007/BF02567629. MR1124311 (93b:14020)

[18] T. SHIFRIN, The osculatory behavior of surfaces in $\mathbb{P}^{5}$, Pacific J. Math. 123 (1986), no. 1, 227256. MR834151 (88h:14041)

[19] A.J. SOMMESE, Hyperplane sections of projective surfaces. I. The adjunction mapping, Duke Math. J. 46 (1979), no. 2, 377-401. http://dx.doi.org/10.1215/S0012-7094-79-04616-7. MR534057 (82f:14033)

[20] A.J. SOmmese and A. VAn DE VEn, On the adjunction mapping, Math. Ann. 278 (1987), no. 1-4, 593-603. http://dx.doi.org/10.1007/BF01458083. MR909240 (88j:14011)

[21] M. SZUREK and J.A. WIŚNIEWSKI, Fano bundles over $\mathbb{P}^{3}$ and $\mathbb{Q}_{3}$, Pacific J. Math. 141 (1990), no. 1, 197-208. MR1028270 (91g:14036)

[22] H. TERAKAWA, The $k$-very ampleness and $k$-spannedness on polarized abelian surfaces, Math. Nachr. 195 (1998), 237-250. http://dx.doi.org/10.1002/mana.19981950113. MR1654705 (2000g:14011)

[23] J.A. WIŚNIEWSKI, Length of extremal rays and generalized adjunction, Math. Z. 200 (1989), no. 3, 409-427. http://dx.doi.org/10.1007/BF01215656. MR978600 (91e:14032)

ANTONIO LANTERI:

Dipartimento di Matematica "F. Enriques"

Università degli Studi di Milano

Via C. Saldini, 50

I-20133 Milano, Italy

E-MAIL: antonio.lanteri@unimi.it

RAQUel MallaVibarRena:

Departamento de Álgebra

Facultad de Ciencias Matemáticas

Universidad Complutense de Madrid

Plaza de las Ciencias, 3

28040 Madrid, Spain

E-MAIL: rmallavi@mat.ucm.es 
RAgni PiEne:

CMA/Department of Mathematics

University of Oslo

P. O. Box 1053 Blindern

NO-0316 Oslo, Norway

E-MAIL: ragnip@math.uio.no

KEY WORDS AND PHRASES: scrolls, inflectional locus, jets, principal parts bundle, Chern classes. 2010 Mathematics Subject Classification: 14C17, 14C20, 14J30, 14 N05.

Received: February 17, 2011. 